\title{
Symbiosis insights through metagenomic analysis of a microbial consortium
}

Tanja Woyke ${ }^{1,2}$, Hanno Teeling ${ }^{3}$, Natalia N. Ivanova ${ }^{1}$, Marcel Hunteman ${ }^{3}$, Michael Richter $^{3}$, Frank Oliver Gloeckner ${ }^{3,4}$, Dario Boffelli ${ }^{1,2}$, Iain J. Anderson ${ }^{1}$, Kerrie W. Barry $^{1}$, Harris J. Shapiro ${ }^{1}$, Ernest Szeto ${ }^{1}$, Nikos C. Kyrpides ${ }^{1}$, Marc Mussmann ${ }^{3}$, Rudolf Amann $^{3}$, Claudia Bergin ${ }^{3}$, Caroline Ruehland ${ }^{3}$, Edward M. Rubin ${ }^{1,2, \dagger} \&$ Nicole Dubilier ${ }^{3, \dagger}$

${ }^{I}$ DOE Joint Genome Institute, Walnut Creek, California 94598, USA.

${ }^{2}$ Lawrence Berkeley National Laboratory, Genomics Division, Berkeley, California 94720, USA.

${ }^{3}$ Max Planck Institute for Marine Microbiology, 28359 Bremen, Germany.

${ }^{4}$ International University Bremen, 28759 Bremen, Germany.

$\dagger$ Correspondence should be addressed to: N. D. (ndubilie@mpi-bremen.de) and E. M. R.

(EMRubin@lbl.gov). 
Symbioses between bacteria and eukaryotes are ubiquitous, yet our understanding of the interactions driving these associations is hampered by our inability to cultivate most host-associated microbes. Here, we used a metagenomic approach to describe four co-occurring symbionts from the marine oligochaete Olavius algarvensis, a worm lacking a mouth, gut, and nephridia. Shotgun sequencing and metabolic pathway reconstruction revealed that the symbionts are sulfur-oxidizing and sulfate-reducing bacteria, all of which are capable of carbon fixation, providing the host with multiple sources of nutrition. Molecular evidence for the uptake and recycling of worm waste products by the symbionts suggests how the worm could eliminate its excretory system, an adaptation unique among annelid worms. We propose a model which describes how the versatile metabolism within this symbiotic consortium provides the host with an optimal energy supply as it shuttles between the upper oxic and lower anoxic coastal sediments which it inhabits. 
Symbiosis plays a major role in shaping the evolution and diversity of eukaryotic organisms ${ }^{1}$. Remarkably, only recently has there been an emerging recognition that most eukaryotic organisms are intimately associated with a complex community of beneficial microbes that are essential for their development, health, and interactions with the environment ${ }^{2}$. This renaissance in symbiosis research stems from advances in molecular approaches that have enabled the study of natural microbial consortia using cultivationindependent methods ${ }^{3-5}$. Metagenomic analyses have provided a new dimension in the study of community organization and metabolism in natural microbial communities ${ }^{6-10}$. To date, however, genomic analyses of symbiotic microbes from eukaryotes have been confined to individual strains (for the only exception see $\mathrm{Wu}$ et $a l .{ }^{11}$ ), limiting our ability to understand the intricate interactions involving communication, competition, and resource partitioning that shape symbiotic microbial communities.

Here, we used random shotgun sequencing and nucleotide-signature based binning to study the symbiotic community in Olavius algarvensis. This marine worm belongs to a group of oligochaetes (phylum Annelida) that lack a mouth, gut, and anus, and are unique among annelid worms in having reduced their nephridial excretory system ${ }^{12}$. They live in obligate and species-specific associations with multiple extracellular bacterial endosymbionts located just below the worm cuticle ${ }^{12}$. Since the symbionts have yet to be grown in culture, their phylogeny has only been accessible through $16 \mathrm{~S}$ rRNA analysis and fluorescence in situ hybridization $(\mathrm{FISH})^{13,14}$. O. algarvensis lives in coastal Mediterranean sediments and harbors a chemoautotrophic sulfur-oxidizing Gammaproteobacterium ( $\gamma 1$ symbiont) and a deltaproteobacterial sulfate reducer $(\delta 1$ symbiont), recently shown to be engaged in an endosymbiotic sulfur cycle ${ }^{14}$. An additional gamma- and deltaproteobacterial symbiont ( $\gamma 3$ and $\delta 4$ symbionts) of unknown function occur consistently in these hosts, and in some individuals a spirochete has been observed as a minor part of the symbiotic consortium (see Supplementary Figure S1a) ${ }^{12}$. 
Given that most chemosynthetic hosts harbor only one or two bacteria, the associations of gutless oligochaetes with multiple symbionts are remarkable, raising a series of questions about their interactions with each other, their host, and the environment. How does the symbiosis compensate for the loss of digestive and excretory systems in the host, what do the various partners gain from this relationship, and is it mutually obligate? What is the selective advantage for $O$. algarvensis in harboring multiple symbiotic partners? Our metagenomic analyses explain how the bacterial consortium meets the energy and waste management needs of its oligochaete host. We describe how resource partitioning between the phylogenetically diverse symbionts benefits both the symbionts and the worm in the heterogeneous environment. Finally, we propose a model showing that the selective advantage of harboring multiple symbionts lies in their ability to supply their host with energy from an abundant and diverse supply of reducing equivalents and electron acceptors as it shuttles between the oxidized and reduced sediment layers.

\section{Metagenomic data analysis and binning}

Pooled samples of 200 O. algarvensis specimens per library were shotgun sequenced (see Supplementary Information, Supplementary Fig. S1b) and the sequences assembled using the whole-genome shotgun assembler JAZZ ${ }^{15}$ (Supplementary Methods, Supplementary Fig. S2). To assign the metagenomic scaffolds to their phylotype origin, we used a combinatory binning approach based on intrinsic DNA signatures (see Supplementary Methods). Binning of the Olavius spp. symbionts' metagenome resulted in the formation of four distinct clusters (Fig. 1). The presence of the corresponding rRNA operons, which represented the only rRNA operons found in these bins and the assembly, enabled us to identify them as the $O$. algarvensis symbionts $\delta 1, \delta 4, \gamma 1$, and $\gamma 3$ (Fig. 1, Supplementary 
Table S1, Supplementary Table S2 providing a comparison to other symbiont genomes). This study illustrates the usefulness of our nucleotide frequency method for the assignment of metagenomic scaffolds from a natural microbial community to a phylotype when using shotgun sequencing. It would not have been possible to reconstruct genome assemblies of the four symbionts based purely on GC contents and scaffold read depth, and without any closely related, fully sequenced reference genome.

Symbiont bin assignments based on 16S rRNA genes were confirmed by phylogenetic analysis of predicted proteins within each cluster of scaffolds (Supplementary Fig. S3), and the clusters were furthermore supported by the distribution of 49 single-copy genes. Although the populations are not clonal, the frequencies of polymorphic sites found in the four symbiont bins, ranging from $0.01-0.1 \%$ (Supplementary Table S4), were rather low compared to environmental microbes such as those from the acid mine drainage ${ }^{8}$. The following discussion describing the metabolism of the symbionts is based on the genes found in each symbiont bin. With a focus on capturing the core metabolic pathways present in the community, it is of minor importance if the genes within each bin originated from a single strain or represent a pangenome of several very closely related strains ${ }^{16}$.

\section{Carbon and energy metabolism}

Gammaproteobacterial symbionts. Chemoautotrophic symbionts feed their hosts by providing them with organic carbon from autotrophic $\mathrm{CO}_{2}$ fixation driven by oxidation of reduced inorganic compounds such as sulfide. In agreement with previous studies indicating the chemoautotrophic, sulfur-oxidizing nature of the O. algarvensis $\gamma 1$ symbiont ${ }^{12}$, our analysis of the $\gamma 1$ bin revealed the presence of genes required for autotrophic $\mathrm{CO}_{2}$ fixation via the Calvin-Benson-Bassham cycle using type I ribulose 1,5- 
bisphosphate carboxylase-oxygenase (RubisCO) $(c b b)$, the oxidation of reduced sulfur compounds (such as $d s r, f c c$ and sox), and the storage of sulfur in globules (sgpB encoding one of three known sulfur globule proteins).

Unexpectedly, our metagenomic analyses revealed, that the $\gamma 3$ symbiont of $O$. algarvensis is also a sulfur-oxidizing chemoautotroph. Several gutless oligochaete species are known to harbor $\gamma 3$ symbionts but the metabolism of these bacteria was previously unknown and the benefit of harboring additional Gammaproteobacteria is unclear. The nearly complete genomic sequence for the $O$. algarvensis $\gamma 3$ symbiont obtained in this study is notable, as this is the first sequenced genome from a chemoautotrophic symbiont. The $\gamma 3$ bin carries all the genes required for a thiotrophic (sulfur-oxidizing) metabolism including those needed for the oxidation of reduced sulfur compounds (including $d s r, a p r, s a t, f c c$, and sox) as well as autotrophic $\mathrm{CO}_{2}$ fixation by means of genes closely related to but phylogenetically distinct from the $\gamma 1$ symbiont (Fig. 2). The absence of sulfur globule proteins in the near complete $\gamma 3$ bin suggests that these symbionts do not store sulfur, supporting transmission electron microscopy analyses showing that only $\gamma 1$ symbionts contain sulfur globules (Giere, pers. communication). In addition to using oxygen as an electron acceptor, the presence of nap and nir gene clusters suggests that the $\gamma 3$ symbionts couple oxidation of reduced sulfur compounds to dissimilatory nitrate reduction under oxygen-limiting conditions (Fig. 2). In deeper sediment layers with neither oxygen nor nitrate both Gammaproteobacteria have the ability to use fumarate as an electron acceptor for the oxidation of reduced sulfur compounds (Fig. 2).

Deltaproteobacterial symbionts. The presence of genes characteristic of dissimilatory sulfate reduction (such as $d s r, q m o$, and $a p r$ ) in both the $\delta 1$ and $\delta 4$ bins suggests that these symbionts are sulfate-reducing bacteria that use oxidized sulfur 
compounds such as sulfate as an electron acceptor, thereby producing sulfide (Fig. 2). In addition to the syntrophic cycling of sulfate and sulfide between the gamma- and deltaproteobacterial $O$. algarvensis symbionts ${ }^{14}$, intermediate sulfur compounds such as tetrathionate and thiosulfate may be cycled between the symbionts. The $\delta 4$ symbiont appears to be able to reduce sulfur compounds of intermediate oxidation states, as suggested by the presence of a multi-heme cytochrome most closely related to tetrathionate reductase of Shewanella oneidensis ${ }^{17}$ and located in a chromosomal cluster with molybdopterin-dependent dehydrogenase related to thiosulfate reductase of Wolinella succinogenes. Cycling of intermediate sulfur compounds is energetically more favorable than the exchange of sulfide and sulfate, as shown previously in experiments with free-living sulfate reducers and sulfur oxidizers ${ }^{18}$.

Heterotrophy is important in sulfate-reducing bacteria and correspondingly we found in the $\delta 1$ bin genes for the transport and utilization of a large variety of carbohydrate substrates, including uronic acids (glucuronate, galacturonate and fructuronate), xylose, fructose, dihydroxyacetone, and polyols (mannitol, sorbitol and glycerol). While all sulfate-reducing bacteria are heterotrophic, only some fix $\mathrm{CO}_{2}$ and it is intriguing that both deltaproteobacterial symbionts are capable of autotrophic carbon fixation via the reductive acetyl-coenzymeA ( $\mathrm{CoA})$ pathway, as well as via the reductive tricarboxylic acid (TCA) cycle (Fig. 2). Thus, O. algarvensis has established an association with four symbionts that are all capable of providing it with organic carbon through three different autotrophic pathways.

One of the most common electron donors for autotrophic sulfate-reducing bacteria is hydrogen. We found gene clusters for periplasmic Ni-Fe hydrogenases, transmembrane high-molecular-weight cytochrome c (hmc) complex, and tetraheam type II tetrahaem cytochrome $c_{3}\left(\mathrm{TpII}-c_{3}\right)$ in the bins of both sulfate-reducing symbionts, as well as TpI-c3 
in the $\delta 1$ bin (Fig. 2). This is a compelling indication for the uptake and oxidation of molecular hydrogen using sulfate as an electron acceptor ${ }^{19}$. It is not clear if hydrogen is provided by the $\gamma$-symbionts. Within the $\gamma 3$ bin, we found genes encoding a pyruvate ferredoxin oxidoreductase (POR), typically used in an alternative route for pyruvate oxidation $^{20}$ and indicative of hydrogen release from low-potential ferredoxins. Released hydrogen could subsequently be taken up by the sulfate-reducing symbionts leading to hydrogen syntrophy within the microbial consortium. Alternative electron donors to hydrogen include glycerol, lactate, proline and betaine, and potentially glycolate and other 2-hydroxy acids, as well as succinate, acetate and propionate.

\section{Symbiont host interactions}

"Feeding" of the host. In other chemosynthetic associations, the symbionts provide their hosts with nutrition using either reduced sulfur compounds or methane as their energy source ${ }^{21}$. In the $O$. algarvensis symbiosis, both reduced sulfur compounds and hydrogen can be used as energy sources, and all four symbionts have the potential to fix $\mathrm{CO}_{2}$ into organic carbon via autotrophy. In addition, the sulfate-reducing symbionts can feed the oligochaete host through heterotrophy by taking up dissolved organic carbon compounds from the environment. Largely all amino acids and a variety of vitamins can be synthesized by the symbionts to provide their host with these required nutrients (Supplementary Table S5). Nutrient transfer to the host is likely to occur via intracellular uptake and digestion of the bacteria, as the number of genes encoding amino acid exporters was not elevated in the symbionts when compared to those of free-living bacteria and the only known family of sugar exporters ${ }^{22}$ was not encoded in any of the symbiont bins. This conclusion is supported by morphological analyses showing symbiont lysis in the basal region of the worm's epidermis ${ }^{23}$. 
Host waste recycling. The reduction of nephridia in the oligochaete host, used for the excretion of nitrogenous waste compounds and osmoregulation, suggests that its symbionts have adapted to carry out these functions. Most aquatic organisms excrete ammonium but urea, which also functions as a common organic osmolyte, has also been found in marine worms with symbionts, such as the hydrothermal vent worm Riftia pachyptila ${ }^{24}$. The genome bins for the $\delta 1, \delta 4$ and $\gamma 3$ symbionts encode a bidirectional uniporter (Amt family transporter ${ }^{25}$ ) for the counter ion-independent and energyindependent uptake of ammonium. A likely urea $\mathrm{ABC}$ transporter for urea uptake is present in the $\gamma 3$ bin, adjacent to an urease operon encoding genes involved in urea hydrolysis. Ammonium and urea uptake by the symbionts would not only aid the host in the removal of these toxic waste products, but also lead to the conservation of valuable nitrogen by the symbionts (Fig. 3).

Marine invertebrates are typical osmoconformers, maintaining their cell volume largely with organic osmoregulatory compounds such as amino acids, taurine, glycine betaine, trimethylamine $N$-oxide (TMAO), and urea, as well as polyols and sugars ${ }^{26}$. The presence of gene clusters encoding proteins used for taurine and glycine betaine import and catabolism in the $\gamma 3$ bin may indicate the use of these osmolytes as both, a carbon and nitrogen source ${ }^{27}$ (Fig. 3). Furthermore, we found pathways for TMAO degradation in the $O$. algarvensis symbionts. Microbial TMAO degradation includes its conversion to trimethylamine by TMAO reductase and further demethylation of trimethylamine either by trimethylamine and dimethylamine dehydrogenases found in Bacteria ${ }^{28}$ or by trimethylamine, dimethylamine and monomethylamine methyltransferases found mostly in Archaea ${ }^{29}$. Genes coding for the key enzymes in both pathways were present in the $\gamma 3$ and $\delta 1$ symbionts, suggestive of their TMAO catabolic activity (Fig. 3). The availability of the osmolyte TMAO would furthermore be particularly advantageous for the sulfur- 
oxidizing symbionts that could use this organic carbon compound as an alternate electron acceptor in the absence of oxygen or nitrate. As just described, the $\gamma 3$ bin encodes enzymes likely to be involved in the reduction of TMAO, although their specificity for this substrate could not be clearly identified.

Polyamines are essential in all organisms for DNA stabilization, DNA replication, and cell proliferation ${ }^{30}$ and represent additional products of host protein breakdown. We found abundant gene clusters encoding $\mathrm{ABC}$ transporters for the uptake of the polyamines putrescine and spermidine in the $\delta 1$ bin, and putrescine in the $\gamma 3$ bin (Fig. 3).

Finally, evidence is provided for the recycling of host fermentation waste products such the dicarboxylate succinate, as well as the monocarboxylates acetate and propionate. Pathways for their utilization and a variety of potential dicarboxylate transporters, and likely monocarboxylate transporters were found in all four symbiont bins. The $\delta 1$ bin encodes 23 tripartite ATP-independent periplasmic (TRAP)-T family dicarboxylate transporters ${ }^{31}$, some of which are likely involved in monocarboxylate and dicarboxylate transport (Fig. 3).

\section{A mutually obligate relationship?}

The lack of a digestive and excretory system in $O$. algarvensis means that its symbionts are crucial for its survival. But is this relationship mutually obligate or can the symbionts survive outside of the host in a free-living stage? Several pieces of evidence from the sequence data and the metabolic reconstruction analyses suggest a lack of evidence for obligate host-dependence of any of the symbionts. This includes the observation that the genomes of the extracellular bacteria do not show AT-bias or genome size reduction (Supplementary Table S2) and there was no obvious loss of essential metabolic pathways 
in the $\delta 1$ or $\gamma 3$ bins suggestive of host-dependence, as is the case for many obligate hostassociated bacteria $^{32,33}$. Finally, we found genes required for cell motility via a flagellum in the $\delta 1, \delta 4$, and $\gamma 3$ bins. While this evidence suggests that the symbionts associated with the worm may have a free-living stage, the presence of a remarkable number of transposases in the $\gamma 3$ and $\gamma 1$ symbiont bins (7.5\% and $20.5 \%$ respectively; Supplementary Tables S4, S6) suggests that these symbionts may be in transition to an obligate symbiotic lifestyle. Bacteria which have recently evolved into obligate symbionts show an increase in frequency of mobile elements, representing a source for chromosomal rearrangements and gene inactivation ${ }^{34}$ (for symbiont transmission hypotheses see Supplementary Information).

\section{From the metagenome to the environment}

The oligochaete symbiosis is inseparably linked to the geochemical properties of the environment, needing access to both reduced and oxidized compounds for energy production and net carbon fixation (Fig 4). In the upper oxic sediment layers of the $O$. algarvensis habitat where no sulfide is present, both the $\gamma 1$ and the $\gamma 3$ symbiont can use reduced sulfur compounds produced internally by the sulfate-reducing symbionts in a syntrophic sulfur cycle. (We have shown previously that the sulfate-reducing symbionts can produce sulfide in $O$. algarvensis under microaerobic conditions comparable to those in the lower oxic zone at $2-5 \mathrm{~cm}$ sediment depth ${ }^{14}$ ). The $\gamma 1$ symbiont can also gain energy independently of the sulfate-reducing symbionts by oxidizing the large supply of sulfur stored in its cytoplasm. For both gammaproteobacterial symbionts, oxygen would be the energetically most favorable electron acceptor (Fig. 4).

As the worm migrates downwards, it encounters sediment layers in which oxygen is no longer present. Under these conditions, the $\gamma 3$ symbiont can use nitrate from the 
environment for the oxidation of reduced sulfur compounds. Given the extremely low concentrations of sulfide in this layer as well as in the deeper reduced sediment layers of the Elba habitat (in the low $\mathrm{nM}$ range ${ }^{14}$ ), it is likely that the sulfate-reducing symbionts provide a large part of the reduced sulfur compounds for the $\gamma$-symbionts under most conditions.

In the deeper sediment layers characterized by reducing conditions and the absence of oxygen or nitrate, hydrogen oxidation by the sulfate-reducing symbionts may occur, in which hydrogen is used as an energy source for the autotrophic fixation of inorganic carbon. As hydrogen concentrations are commonly very low in most marine sediments, heterotrophic pathways should also play an important role for the deltaproteobacterial symbionts under these reducing conditions. Although energetically less favorable than nitrate or oxygen, organic electron acceptors including TMAO (host-derived) and fumarate (produced by the $\delta$-symbionts) are provided internally within the symbiosis and may be used by the $\gamma$-symbionts for the oxidation of reduced sulfur compounds. This would enable the $\gamma 1$ symbiont to replenish its sulfur stores, which could be fully oxidized using the energetically more favorable electron acceptor oxygen when the worm returns to the oxic zone. Fumarate respiration by the $\gamma$-symbionts would produce succinate, which could be used by the deltaproeobacterial symbionts as an energy source and reoxidized to fumarate, thus leading to a syntrophic cycling of reductants and oxidants.

Our analysis of the O. algarvensis microbial genomes has provided insights on how resources are used and shared among the different symbionts and with their host, and how different metabolic pathways are used by the symbionts to generate energy as the worm migrates through the chemocline. We have shown how the O. algarvensis symbiosis is unique among known chemosynthetic symbioses, as reductants and oxidants are not only supplied from the environment but also internally to drive energy production. 
Thus, this comprehensive metagenomic analysis shows that these highly integrated synergistic assemblages of multiple bacterial partners provide their eukaryotic host with an optimal energy supply and waste management through resource partitioning and cooperation during syntrophic cycling of oxidized and reduced compounds. 


\section{Methods}

O. algarvensis specimens were collected off Capo di Sant' Andrea, Elba, Italy. Metagenomic libraries were constructed from 200 pooled $O$. algarvensis specimens per library. A small insert pMCL200 library was made from a nycodenz-separated, symbiont-enriched sample and two pCC1Fos fosmid libraries were constructed using the CopyControl $^{\mathrm{TM}}$ Fosmid Library Construction Kit (Epicentre). 16S rRNA PCR libraries were created from the DNA sources used for each of the libraries and approximately 384 clones sequenced and analyzed. From the shotgun libraries, we created $204 \mathrm{Mb}$ of vectorand quality-trimmed sequence. This data was assembled using JAZZ, resulting in a set of 2,286 scaffolds, which were binned using a combinatory approach based on dimer to hexamer frequencies using the newly developed program $^{35}$. Final clusters with 511 scaffolds were verified by phylogenetic affiliation of each scaffold based on the most common phylogeny of its predicted proteins, by a Bayesian classifier, and by checking for paralogs of 49 genes that typically occur with only one copy per genome (Kunin et al, unpublished). To assess nucleotide sequence variation within the bins, we analyzed the multiple alignment of the JAZZ assembly. Potential open reading frames (ORFs) were identified using "mORFind" (Waldmann, unpublished) and annotation performed with the GenDB v2.2 system $^{36}$ and MicHanThi ${ }^{37}$. The annotated symbiont metagenome was loaded into the metagenomics version of Integrated Microbial Genomes/M (IMG/M) ${ }^{38}$ (http://img.jgi.doe.gov/m).

Details for all methods used are provided in the Supplementary Information. 


\section{References}

1. Margulis, L. Symbiosis in Cell Evolution (W. H. Freeman, New York, 1993).

2. Ruby, E. G., Henderson, B. \& McFall-Ngai, M. Microbiology. We get by with a little help from our (little) friends. Science 303, 1305-7 (2004).

3. DeLong, E. F. Microbial population genomics and ecology. Curr. Opin. Microbiol. 5, 520-4 (2002).

4. Handelsman, J. Metagenomics: application of genomics to uncultured microorganisms. Microbiol. Mol. Biol. Rev. 68, 669-85 (2004).

5. Riesenfeld, C. S., Schloss, P. D. \& Handelsman, J. Metagenomics: genomic analysis of microbial communities. Аnпи. Rev. Genet. 38, 525-52 (2004).

6. DeLong, E. F. et al. Community genomics among stratified microbial assemblages in the ocean's interior. Science 311, 496-503 (2006).

7. Hallam, S. J. et al. Reverse methanogenesis: testing the hypothesis with environmental genomics. Science 305, 1457-62 (2004).

8. Tyson, G. W. et al. Community structure and metabolism through reconstruction of microbial genomes from the environment. Nature 428, 37-43 (2004).

9. Venter, J. C. et al. Environmental genome shotgun sequencing of the Sargasso Sea. Science 304, 66-74 (2004).

10. Tringe, S. G. et al. Comparative metagenomics of microbial communities. Science 308, 554-7 (2005).

11. Wu, D. et al. Metabolic Complementarity and genomics of the dual bacterial symbiosis of sharpshooters. PLoS Biol. 4, e188 (2006).

12. Dubilier, N., Blazejak, A. \& Ruhland, C. Symbioses between bacteria and gutless marine oligochaetes. Prog. Mol. Subcell. Biol. 41, $251-75$ (2006). 
13. Blazejak, A., Erseus, C., Amann, R. \& Dubilier, N. Coexistence of bacterial sulfide oxidizers, sulfate reducers, and spirochetes in a gutless worm (Oligochaeta) from the Peru margin. Appl. Environ. Microbiol. 71, 1553-61 (2005).

14. Dubilier, N. et al. Endosymbiotic sulphate-reducing and sulphide-oxidizing bacteria in an oligochaete worm. Nature 411, 298-302 (2001).

15. Aparicio, S. et al. Whole-genome shotgun assembly and analysis of the genome of Fugu rubripes. Science 297, 1301-10 (2002).

16. Medini, D., Donati, C., Tettelin, H., Masignani, V. \& Rappuoli, R. The microbial pan-genome. Curr. Opin. Genet. Dev. 15, 589-94 (2005).

17. Mowat, C. G. et al. Octaheme tetrathionate reductase is a respiratory enzyme with novel heme ligation. Nat. Struct. Mol. Biol. 11, 1023-4 (2004).

18. van den Ende, F. P., Meier, J. \& van Gemerden, H. Syntrophic growth of sulfatereducing bacteria and colorless sulfur bacteria during oxygen limitation. FEMS Microbiol. Ecol. 23, 65-80 (1997).

19. Matias, P. M., Pereira, I. A., Soares, C. M. \& Carrondo, M. A. Sulphate respiration from hydrogen in Desulfovibrio bacteria: a structural biology overview. Prog. Biophys. Mol. Biol. 89, 292-329 (2005).

20. Kletzin, A. \& Adams, M. W. Molecular and phylogenetic characterization of pyruvate and 2-ketoisovalerate ferredoxin oxidoreductases from Pyrococcus furiosus and pyruvate ferredoxin oxidoreductase from Thermotoga maritima. J. Bacteriol. 178, 248-57 (1996).

21. Cavanaugh, C. M., McKiness, Z. P., Newton, I. L. G. \& Stewart, F. J. Marine chemosynthetic symbioses. In The Prokaryotes: A handbook on the biology of bacteria (eds. Dworkin, M. et al.) (Springer Verlag, New York, 2006). 
22. Liu, J. Y., Miller, P. F., Gosink, M. \& Olson, E. R. The identification of a new family of sugar efflux pumps in Escherichia coli. Mol. Microbiol. 31, 1845-51 (1999).

23. Giere, O. \& Erseus, C. Taxonomy and new bacterial symbioses of gutless marine Tubificidae (Annelida, Oligochaete) from the Island of Elba (Italy). Org. Divers. Evol. 2, 289-297 (2002).

24. De Cian, M., Regnault, M. \& Lallier, F. H. Nitrogen metabolites and related enzymatic activities in the body fluids and tissues of the hydrothermal vent tubeworm Riftia pachyptila. J. Exp. Biol. 203, 2907-20 (2000).

25. Khademi, S. et al. Mechanism of ammonia transport by Amt/MEP/Rh: structure of AmtB at 1.35 A. Science 305, 1587-94 (2004).

26. Yancey, P. H., Blake, W. R. \& Conley, J. Unusual organic osmolytes in deep-sea animals: adaptations to hydrostatic pressure and other perturbants. Comp. Biochem. Physiol. Part A Mol. Integr. Physiol. 133, 667-76 (2002).

27. Denger, K., Ruff, J., Schleheck, D. \& Cook, A. M. Rhodococcus opacus expresses the xsc gene to utilize taurine as a carbon source or as a nitrogen source but not as a sulfur source. Microbiology 150, 1859-67 (2004).

28. Yang, C. C., Packman, L. C. \& Scrutton, N. S. The primary structure of Hyphomicrobium X dimethylamine dehydrogenase. Relationship to trimethylamine dehydrogenase and implications for substrate recognition. Eur. J. Biochem. 232, 264-71 (1995).

29. Paul, L., Ferguson, D. J., Jr. \& Krzycki, J. A. The trimethylamine methyltransferase gene and multiple dimethylamine methyltransferase genes of Methanosarcina barkeri contain in-frame and read-through amber codons. J. Bacteriol. 182, 2520-9 (2000). 
30. Cohen, S. S. A Guide to the Polyamines (Oxford University Press, New York, 1998).

31. Kelly, D. J. \& Thomas, G. H. The tripartite ATP-independent periplasmic (TRAP) transporters of bacteria and archaea. FEMS Microbiol. Rev. 25, 405-24 (2001).

32. Moran, N. A. Microbial minimalism: genome reduction in bacterial pathogens. Cell 108, 583-6 (2002).

33. Moran, N. A. Tracing the evolution of gene loss in obligate bacterial symbionts. Curr. Opin. Microbiol. 6, 512-8 (2003).

34. Moran, N. A. \& Plague, G. R. Genomic changes following host restriction in bacteria. Curr. Opin. Genet. Dev. 14, 627-33 (2004).

35. Huntemann, M. MetaClust - Entwicklung eines modularen Programms zum Clustern von Metagenomfragmenten anhand verschiedener intrinsischer DNASignaturen. Thesis, University Bremen (2006).

36. Meyer, F. et al. GenDB--an open source genome annotation system for prokaryote genomes. Nucleic Acids Res. 31, 2187-95 (2003).

37. Quast, C. MicHanThi - Design and Implementation of a System for the Prediction of Gene Functions in Genome Annotation Projects. Thesis, University Bremen (2006).

38. Markowitz, V. M. et al. The integrated microbial genomes (IMG) system. Nucleic Acids Res. 34, D344-8 (2006).

39. Teeling, H., Waldmann, J., Lombardot, T., Bauer, M. \& Glockner, F. O. TETRA: a web-service and a stand-alone program for the analysis and comparison of tetranucleotide usage patterns in DNA sequences. BMC Bioinformatics 5, 163 (2004). 
Supplementary Information is linked to the online version of the paper at www.nature.com/nature.

Acknowledgements This work was performed under the auspices of the US Department of Energy's Office of Science, Biological and Environmental Research Program, and by the University of California, Lawrence Livermore National Laboratory under Contract No. W-7405-Eng-48, Lawrence Berkeley National Laboratory under contract No. DE-AC02-05CH11231 and Los Alamos National Laboratory under contract No. DE-AC02-06NA25396 and the Max Planck Society. We thank members of the Rubin, Jim Bristow and Phil Hugenholtz labs as well as the JGI for their contributions. We furthermore thank Victor Markowitz, Eileen Dalin, Nik Putnam, Rotem Sorek, Tijana Glavina del Rio, Asaf Salamov, Art Kobayashi, and Kennan Kellaris for their assistance. At the Max Planck Institute for Marine Microbiology we thank Silke Wetzel for excellent technical assistance. We are grateful to Christian Lott and the staff of the HYDRA field station at Elba for their generous support and help in sampling the worms. Assembled sequences from the Olavius spp. symbionts' metagenome have been deposited into the NCBI database under the project accession AASZ00000000. The annotated Olavius spp. symbionts' bins were incorporated into the metagenomics version of the U.S. Department of Energy Joint Genome Institute Integrated Microbial Genomes (IMG), IMG/M (http://img.jgi.doe.gov/m).

Author information Correspondence and requests for materials should be addressed to: N. D. (ndubilie@mpi-bremen.de) and E. M. R. (EMRubin@lbl.gov). 
Figure 1

Clustering of the symbiont scaffolds. Visualization of the first three components of a principal component analysis, in which GC-content, net read depth, z-scores for all possible 64 trinucleotides and 256 tetranucleotides were incorporated with equal weight (z-scores calculated with TETRA ${ }^{39}$, normalized by length). The colors represent the four clusters of scaffolds (calculated with MetaClust), that were binned based on GC-content, dinucleotide relative abundance, Markov model-based statistical evaluations of tri-, tetra and pentamer over- and underrepresentation and normalized chaos game representations for tri- to hexamers; sequences $<5 \mathrm{~kb}$ are not represented. Scaffolds containing $16 \mathrm{~S}$ rRNA genes are tagged.

Figure 2

Reconstruction of the symbionts' physiology. PHA, polyhydroxyalkanoates. CM, cell material EMP, Embden-Meyerhof pathway. TCA, tricyclic acid. C-taxis, chemotaxis. APS, adenosine 5'-phosphosufate. G 3-P, glyceraldehyde 3phosphate. Hmc, high-molecular-weight cytochrome c. Tpl/II- $c_{3}$, tetrahaem type I/II tetrahaem cytochrome $\mathrm{c}_{3} . \mathrm{H}_{2}$ ase, hydrogenase. PEP, phosphoenolpyruvatye. CoA, coenzymeA. ?, indicates the lack of nitric oxide reductase in the $\mathrm{\gamma} 3$ genome bin. TRAP, tripartite ATP-independent periplasmic. Numbers in parenthesis indicate the numbers of amino acids/vitamin biosynthesis pathways found (Supplementary Table S5).

Figure 3 
Reconstruction of symbiont host interactions. The metagenomic data uncovered pathways for the uptake and recycling of organic osmolytes and excretion products of the worm by its symbionts. TMAO, trimethylamine $\mathrm{N}$-oxide. TMA, trimethylamine.

Figure 4

Model for energy metabolism in the symbiosis. O. algarvensis inhabits shallow Mediterranean ocean sediments (5-15 cm depth). Electron acceptors and donors are available to the symbionts in all sediment layers, with some supplied from the environment (shown in the box and triangles) and some internally (shown next to the worms). Carbon is gained autotrophically using reduced sulfur compounds ( $\mathrm{\gamma}$-symbionts) or hydrogen ( $\delta$-symbionts) as well as heterotrophically ( $\delta$-symbionts). $\mathrm{cms}=\mathrm{cm}$ below sea floor. SRS, sulfate-reducing symbionts. SOS, sulfur-oxidizing symbionts. TMAO, trimethylamine $\mathrm{N}$-oxide. AM, anaerobic metabolites. $S_{\text {red, }}$ reduced sulfur compounds. $S_{\text {ox }}$, oxidized sulfur compounds. OrgC, organic compounds. 


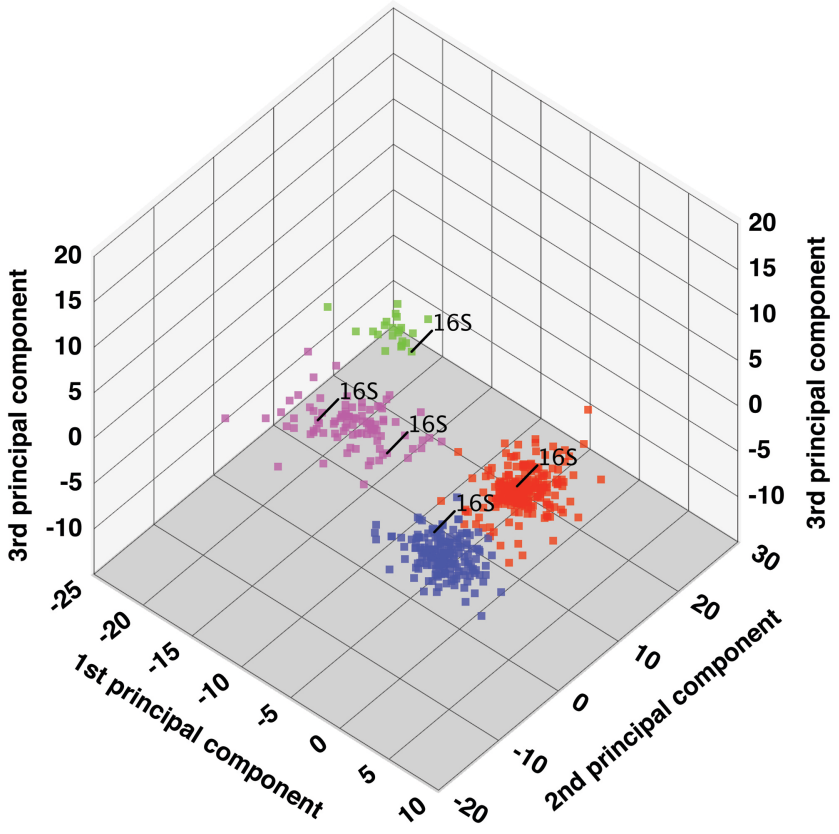

O. algarvensis $\delta 1$ symbiont

0 . algarvensis $\delta 4$ symbiont

$O$. algarvensis $\gamma 1$ symbiont

O. algarvensis $\gamma 3$ symbiont 


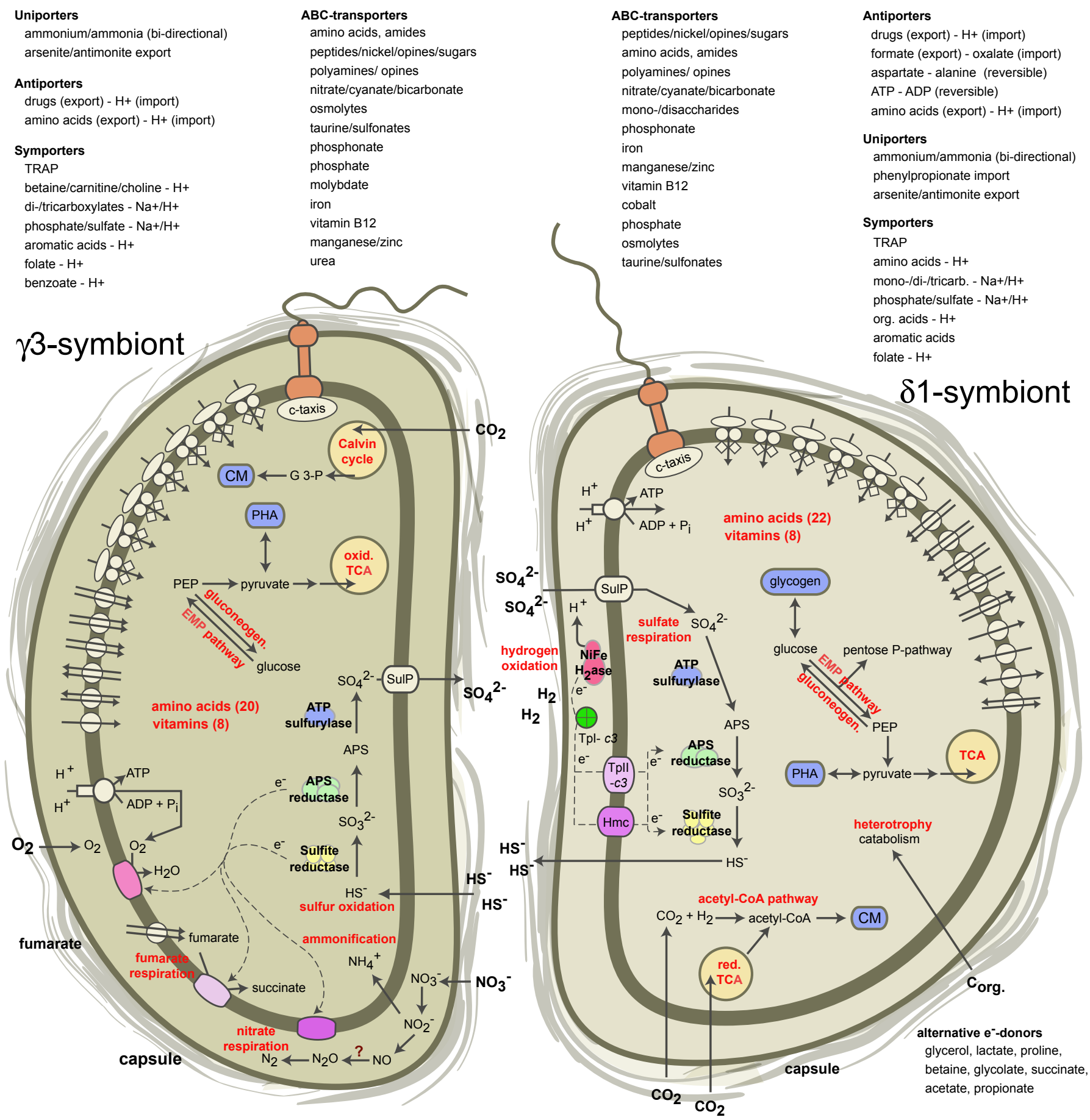




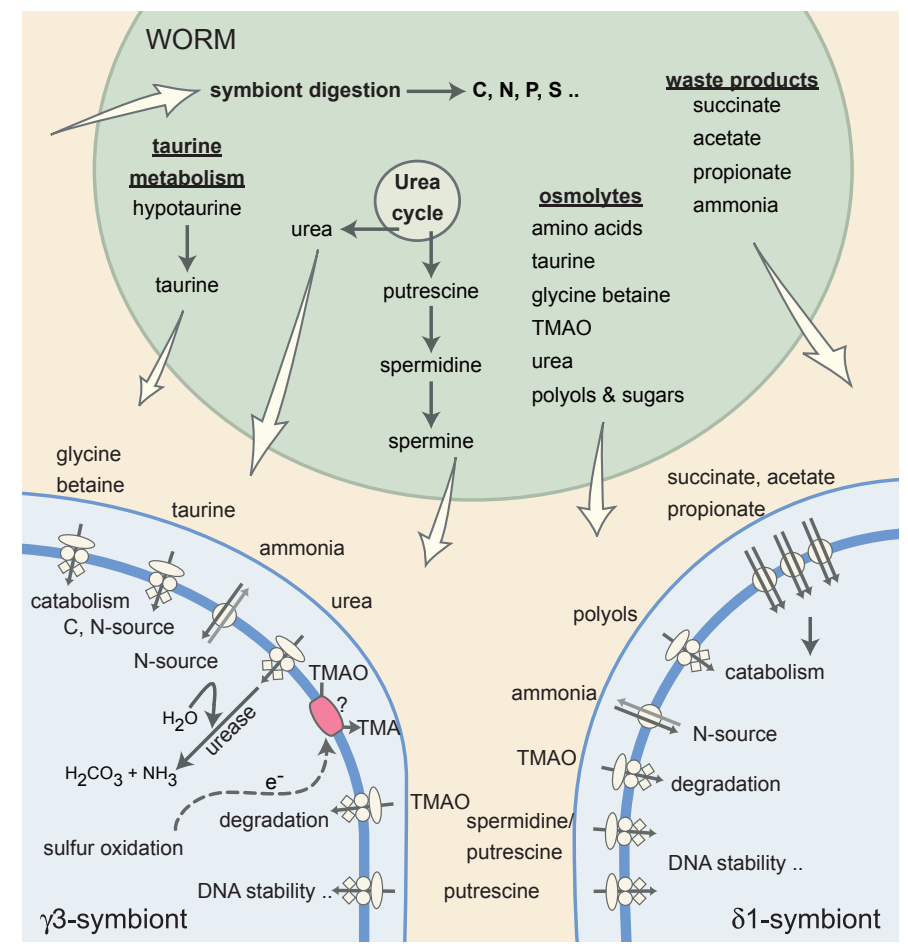




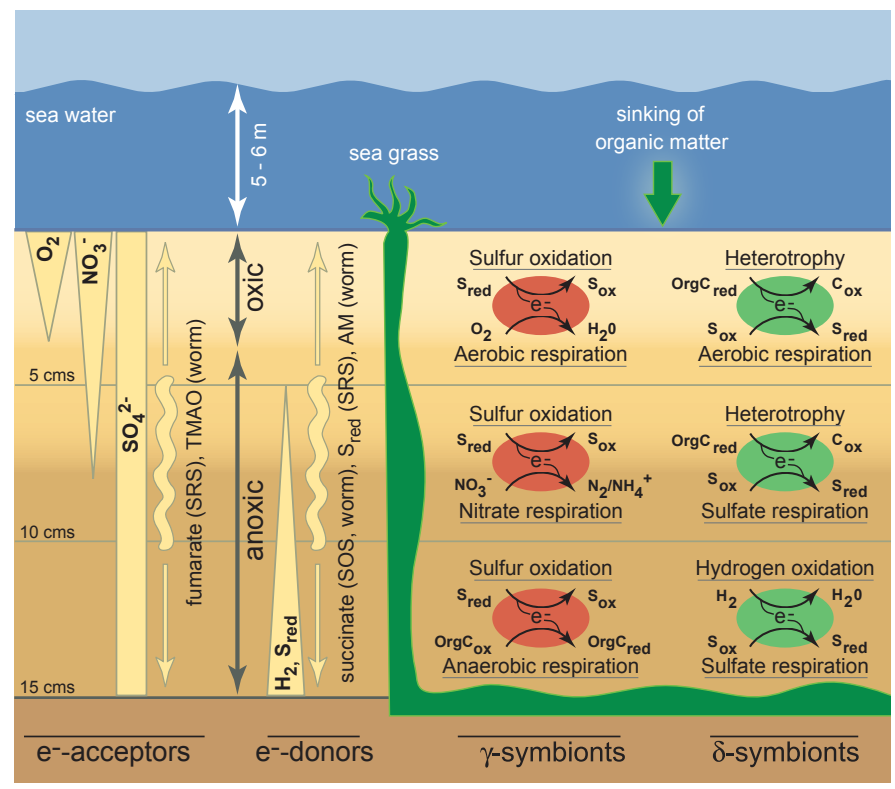




\title{
Symbiosis insights through metagenomic analysis of a microbial consortium
}

\author{
Supplementary Information
}

\section{Supplementary Methods}

\section{Specimen collection}

Juvenile and adult Olavius algarvensis specimens were collected in May and September 2004 from $5.6 \mathrm{~m}$ water depth in silicate sediments around sea grass beds of Posidonia oceanica in a bay off Capo di Sant' Andrea, Elba, Italy (42 48'26"N, 01008'28"E). The worms were removed from the sediment via decantation with seawater and identified under a dissection scope. Fresh samples collected in September 2004 were kept in the original sediment and seawater for 3 days until preparation. All other specimens were cleaned by successive washes in sterile seawater, snap-frozen on dry ice and stored at $80^{\circ} \mathrm{C}$ until further processing. O. algarvensis is the dominant species at the collection site, however an additional gutless oligochaete species named $O$. ilvae co-occurs ${ }^{1}$. Identification at the species level requires careful examination of individual sexually mature worms by an expert and was thus not possible for this study.

\section{Bacterial symbiont enrichment for the $3 \mathrm{~kb}$ library}

Bacterial cells from approximately 200 live worms ( 180 mg of sample containing $\sim 2 \mathrm{x}$ $10^{8}$ bacteria) were enriched using discontinuous nycodenz density gradient centrifugation. Briefly, fresh worms were removed from the sediment via decantation, cleaned by successive washes in sterile seawater and gently homogenized in $2 \mathrm{ml}$ phosphate-buffered saline (PBS), pH 7.4 using a glass dounce homogenizer. A step gradient of 1.146-1.083 g/ml density was prepared with Histodenz ${ }^{\mathrm{TM}}$ (Sigma, St. Louis, $\mathrm{MO}$ ) in $5 \mathrm{ml}$ OpiSeal Polyallomer tubes (Beckman Coulter, Fullerton, CA) and the cell suspension loaded on top of the gradient. The overlain gradient was then centrifuged at $10,000 \mathrm{~g}$ in a Beckman L8-M ultracentrifuge and SW 55Ti swing rotor for $1 \mathrm{~h}$ at $4^{\circ} \mathrm{C}$. Following centrifugation, 200-300 $\mu \mathrm{l}$ fractions were withdrawn from the bottom of the 
gradient tube and diluted with 10 vol of PBS to remove excess nycodenz. Cells were pelleted and resuspended in PBS and fractions evaluated semi-quantitatively for the enrichment of bacterial cells using real-time PCR amplification of 16S and 18S rRNA genes. As expected, the best enrichment of bacterial cells was found in higher density fractions, which were subsequently used for DNA extraction.

\section{DNA extraction}

For the fosmid libraries, metagenomic high molecular weight (HMW) DNA was extracted from approximately 200 pooled frozen worms for each library. The frozen worms were ground into fine powder under liquid nitrogen with mortar and pestle, transferred to a screw-cap tube and DNA extracted using the Qiagen Genomic-tip (Qiagen, Valencia, CA) according to the manufacturer's instructions. Approximately 60 $\mu \mathrm{g}$ of HMW metagenomic DNA was purified per 200 frozen specimens. For the $3 \mathrm{~kb}$ library, metagenomic DNA was extracted from nycodenz gradient enriched bacterial cells using Bactozol ${ }^{\mathrm{TM}}$ (Molecular Research Center, Inc., Cincinnati, $\mathrm{OH}$ ) according to the manufacturer. Fresh cells recovered from three combined nycodenz fractions yielded $\sim 400 \mathrm{ng}$ of DNA. DNA concentrations and purities were assessed by agarose gel electrophoresis and spectrophotometric analysis.

\section{Shotgun library construction \& end-sequencing}

Three metagenomic shotgun libraries were constructed for this study: one $3 \mathrm{~kb}$ library from live worms collected in September 2004 and two fosmid libraries from frozen worms collected in April 2004 and September 2004.

$3 \mathrm{~kb}$ library. A small insert library was constructed from DNA derived from the nycodenz-enriched sample collected in September 2004. Briefly, $300 \mathrm{ng}$ of metagenomic DNA was randomly sheared to $2-4 \mathrm{~kb}$ fragments using a HydroShear (GeneMachines, San Carlos, CA). The sheared DNA was separated on an agarose gel, gel-purified using the QIAquick Gel Extraction Kit and end-repaired using the End-it ${ }^{\mathrm{TM}}$ DNA End-Repair kit (Epicentre, Madison, WI) according to the manufacturer's instructions. After an additional agarose gel separation, 2-4 kb DNA fragments were gel-purified once more. 
The entire DNA extract was blunt-end ligated into $100 \mathrm{ng}$ of pMCL200 vector $\mathrm{O} / \mathrm{N}$ at $16^{\circ} \mathrm{C}$ using T4 DNA ligase (Roche Applied Science, Indianapolis, IN) and 10\% (vol/vol) polyethylene glycol (Sigma). The ligation was phenol-chloroform extracted, ethanol precipitated and resuspended in $15 \mu \mathrm{T}$ TE. According to the manufacturer's instructions, 1 $\mu 1$ of ligation product was electroporated into ElectroMAX DH10B ${ }^{\mathrm{TM}}$ Cells (Invitrogen, Carlsbad, CA) and plated on selective agar plates. Positive library clones were picked using the Q-Bot multitasking robot (Genetix, Dorset, U.K.) and grown in selective media for sequencing.

Fosmid libraries. Fosmid libraries were constructed using the CopyControl ${ }^{\mathrm{TM}}$ Fosmid Library Production Kit (Epicentre). Briefly, $\sim 20 \mu \mathrm{g}$ of metagenomic DNA derived from the frozen samples was randomly sheared using a HydroShear, blunt-end repaired as described above and separated on an agarose pulse-field gel $\mathrm{O} / \mathrm{N}$ at $4.5 \mathrm{~V} / \mathrm{cm}$. The $35 \mathrm{~kb}$ fragments were excised, gel-purified using $\operatorname{AgarACE}^{\mathrm{TM}}$ (Promega, Madison, WI) digestion, followed by phenol-chloroform extraction, and ethanol precipitation. DNA fragments were ligated into the $\mathbf{p C C 1 F o s}{ }^{\mathrm{TM}}$ Vector. The ligation was packaged using MaxPlax ${ }^{\mathrm{TM}}$ Lambda Packaging Extract and used to transfect TransforMax ${ }^{\mathrm{TM}}$ EPI300 Escherichia coli. Transfected cells were plated on selective agar plates and fosmid clones picked using the Q-Bot multitasking robot and grown in selective media for sequencing.

End-sequencing. Plasmids were amplified using the TempliPhi ${ }^{\mathrm{TM}}$ DNA Sequencing Template Amplification Kit (Amersham Biosciences, Piscataway, NJ) and sequenced using standard M13 -28 or -40 primers and the BigDye sequencing kit (Applied Biosystems, Foster City, CA) according to the manufacturer's instructions. The reactions were purified using magnetic beads and run on an ABI PRISM 3730 (Applied Biosystems) capillary DNA sequencer (for research protocols, see www.jgi.doe.gov).

\section{S rRNA libraries \& phylogenetic analysis}

16S rRNA PCR libraries were created from DNA sources used for all three metagenomic libraries. Amplification of $16 \mathrm{~S}$ rRNA genes was performed using the bacteria-specific universal primers 27f (5'-AGAGTTTGATCCTGGCTCAG-3') and 1492r (5'- 
GGTTACCTTGTTACGACTT-3' ${ }^{2}$. The following cycling conditions were used: $94^{\circ} \mathrm{C}$ for $5 \mathrm{~min}$, followed by 20 cycles of $94^{\circ} \mathrm{C}$ for $30 \mathrm{sec}, 55^{\circ} \mathrm{C}$ for $25 \mathrm{sec}, 72^{\circ} \mathrm{C}$ for $90 \mathrm{sec}$, and an extension at $72^{\circ} \mathrm{C}$ for $7 \mathrm{~min}$. To minimize heteroduplex formation, a reconditioning step was applied ${ }^{3}$. Briefly, PCR reactions were diluted 10-fold into fresh reaction mixtures of the same composition and cycled three more times using the above parameters. PCR products of five replicate reactions were combined, gel-extracted as described above and ligated into the pCR4-TOPO vector using the TOPO TA Cloning Kit (Invitrogen). Ligations were then electroporated into One Shot TOP10 Electrocomp ${ }^{\mathrm{TM}}$ E. coli cells and plated on selective media agar plates. Approximately 384 clones per library were picked and grown in selective media for sequencing (see above).

The bi-directional 16S rRNA gene sequence reads were end-paired and trimmed for PCR primer sequence and quality. Approximately 3\% of the sequences were removed as putative chimeras by identification with Bellerophon ${ }^{4}$. The resulting chimera-free sequences were evaluated using BLAST analysis ${ }^{5}$ against sequences in the NCBI database and the $16 \mathrm{~S}$ rRNA sequences of the $O$. ilvae and O. algarvensis symbionts (unpublished data). Phylogenetic trees were calculated by neighbor joining analyses using the ARB software package (www.arb-home.de) ${ }^{6}$. Only sequences $\geq 1,400$ bp were used for tree construction.

\section{Processing, analysis \& assembly of shotgun data}

Initial data set. The initial data set was derived from the three shotgun libraries described above. We sequenced 279,157 reads from the $3 \mathrm{~kb}$ library, containing $279 \mathrm{Mb}$ of raw sequence. 36,095 reads were sequenced from the two $35 \mathrm{~kb}$ libraries, containing $37 \mathrm{Mb}$ of raw sequence. The reads were screened for vector using cross_match, then trimmed for vector and quality ${ }^{7}$. Reads $<100$ bases after trimming were excluded. This reduced the amount of data to 250,034 reads $(185 \mathrm{Mb})$ of $3 \mathrm{~kb}$ library end-sequence, and 31,414 reads $(19 \mathrm{Mb})$ of $35 \mathrm{~kb}$ library end-sequence.

Analysis of unassembled shotgun sequences. Unassembled shotgun sequence reads (trimmed for vector sequence and quality) were evaluated using BLAST analysis ${ }^{5}$ against the NCBI nr database (BLASTx, e-value 1e-3) and NCBI nt database (BLASTn), as well 
as the $16 \mathrm{~S}$ rRNA gene sequences of the $O$. ilvae and $O$. algarvensis symbionts (BLASTn).

Jazz assembly parameters. The data was assembled using release 2.8 of JAZZ, a WGS assembler developed at the $\mathrm{JGI}^{7,8}$. A word size of 13 was used for seeding alignments between reads. The unhashability threshold was set to 50, preventing words present in more than 50 copies in the data set from being used to seed alignments. A mismatch penalty of -30.0 was used, which will generally assemble sequences that are more than about $97 \%$ identical. As the different organisms in the data set were expected to be present at different sequence depths, the usual depth-based bonus/penalty system was turned off.

Post-assembly analysis. The initial assembly contained 5,016 scaffolds, with $42 \mathrm{Mb}$ of sequence, of which $37 \%$ were gaps. As JAZZ links contigs into scaffolds based on fosmid paired-end information and pads these gaps with N's based on the known approximate insert fosmid size, many scaffolds are gapped. The scaffold N/L50 was $122 / 73 \mathrm{~kb}$, while the contig N/L50 was $741 / 8.2 \mathrm{~kb}$. If the scaffolds are sorted by total length in descending order, the scaffold N50 value is equal to the number of scaffolds one needs to go down the list before one has encompassed half of the total scaffold sequence in the set. The scaffold L50 value is then the total length of the smallest scaffold in the "top half" of this list. The Contig N50 and L50 are values analogous to the scaffold N50 and L50 values with the difference that the contig values are calculated using net, instead of total, scaffold lengths. Redundant scaffolds were identified by aligning all scaffolds with less than $5 \mathrm{~kb}$ of contig sequence against those with more than $5 \mathrm{~kb}$ of contig sequence using BLAST-like alignment tool ${ }^{9}$. Any scaffolds from the former set that matched any of the larger over more than $80 \%$ of their length were excluded. Short scaffolds $(<1 \mathrm{~kb}$ of contig sequence) were also excluded. The unassembled reads and short scaffolds largely represent the lower abundance species as well as worm DNA within this environmental sample, as suggested by the presence of the metagenomic reads encoding the $O$. ilvae symbionts $16 \mathrm{~S}$ rRNA genes within this shrapnel. The filtering left 2,286 scaffolds, with $39 \mathrm{Mb}$ of sequence, of which 40\% was gap. The scaffold N/L50 
was $106 / 80 \mathrm{~kb}$, while the contig N/L50 was 606/9.6 kb. Approximately $61 \%$ of the reads fell into the filtered assembly. This filtered scaffold set served as the starting point of all downstream analyses.

\section{Binning}

Scaffolds from the Olavius spp. symbionts' metagenome were binned by a combinatory approach based on the following intrinsic DNA signatures: (a) GC-content (b) dinucleotide relative abundance ${ }^{10}$ (c) Markov model-based statistical evaluations of tri-, tetra and pentamer over- and under-representation ${ }^{11}$ and (d) normalized chaos game representations for tri- to hexamers Deschavanne ${ }^{12,13}$. Values for (c) and (d) were computed by ocount and cgr, two self-written C-programs that are available from www.megx.net/tetra.

A self-written Java program (MetaClust ${ }^{14}$ ) was used to automatically trigger the individual calculations and subsequently store them in a MySQL database. Seven different combinations of subsets of the individual methods were built for all scaffolds exceeding $50 \mathrm{~kb}$ and imported into Cluster $3.0^{15}$. The data was normalized and a hierarchical clustering was computed using complete linkage and the Euclidian distance as distance measure. The corresponding result files were analyzed using Java TreeView (http://genetics.stanford.edu/ alok/TreeView/) and merged into consensus clusters in a semi-automatic manner by parsing the Java TreeView result files. This procedure was repeated for all scaffolds exceeding $15 \mathrm{~kb}$ and thereafter for all scaffolds exceeding $5 \mathrm{~kb}$. Shorter scaffolds were discarded because (a) the reliability of signature-based scaffold affiliation declines with decreasing sequence lengths and (b) the fraction of short unassembled scaffolds contain chimeric sequences. After each of these steps, the newer and the former clusters were compared and ambiguous scaffolds were sorted out. A Bayesian classifier ${ }^{16}$ was trained with all scaffolds $\geq 50 \mathrm{~kb}$ and subsequently used to assign some of the much shorter scaffolds with ambiguous classifications. Final clusters were verified threefold, (a) by phylogenetic affiliation of each scaffold based on the most common phylogeny of its predicted proteins (BLASTp, e-value $\geq 1 \mathrm{e}-5$, NCBI nr) (b) by a Bayesian classifier and (c) by checking for paralogs of 49 genes that typically occur with only one copy per genome (Kunin et al, unpublished). 
Despite all the support for our binning, it is noteworthy that the approach is statistical by nature and thus the bins likely do contain false positive. It should also be stressed that the binning-approach benefits from a low diversity and a confined habitat of the to be separated organisms, since successful separation becomes the more problematic the higher the diversity of a sample gets. Low-diversity symbiotic communities are optimal samples in this regard, although their assembly and binning can be affected by an elevated proportion of lateral gene transfer among the symbionts.

\section{Gene prediction and annotation}

Potential open reading frames (ORFs) were identified using the meta gene prediction software "mORFind" (Waldmann, unpublished) developed at the MPI-Bremen. This system analyzes and combines the output of the three commonly used gene-finders CRITICA $^{17}$, GLIMMER $^{18}$ and ZCURVE $^{19}$ to enhance sensitivity and specificity. To resolve conflicts, an iterative post-processing algorithm is used taking into account signal peptide $^{20}$ and transmembrane ${ }^{21}$ predictions, ORF-length, and the number of gene-finders by which an ORF has been predicted. The system was adapted to deal with typical problems of community sequencing projects like ambiguities, stretches of Ns, and fragmented genes. Annotation was performed with the GenDB v2.2 system $^{22}$, seeking for each predicted ORF observations from similarity searches against sequence databases (nr, Swiss-Prot, Kegg-Genes, release December 2005) and protein family databases (Pfam (release 19.0), InterPro (release 12.0, InterProScan version 4.2)), and from predictive signal peptide- (SignalP v3.0 ${ }^{20}$ ) and transmembrane helix-analysis (TMHMM v2.0 ${ }^{21}$ ). tRNA genes were identified using tRNAScan-SE ${ }^{23}$ and rRNA genes were detected by standard similarity searches $\left(\mathrm{BLAST}^{5}\right.$ ) against public nucleotide databases and the $16 \mathrm{~S}$ rRNA sequences of the $O$. ilvae and $O$. algarvensis symbionts. Predicted protein coding sequences were automatically annotated with the software MicHanThi ${ }^{24}$ developed at the MPI Bremen. The system simulates the human annotation process using fuzzy logic. First, informative BLAST observations are selected taking into account several BLAST parameters. The gene product is then assembled by functional clustering of observations and by selection of the most supported one. Each annotation is labelled by the corresponding reliability value to support further human inspection. Once the gene 
product is set, MicHanThi adds further information like gene name, EC, and GO numbers to each protein coding gene based on Swiss-Prot and InterPro observations, respectively. A functional classification was performed with similarity searches against $\mathrm{COG} v 2^{25}$. All ORFs described in this publication were manually refined. All binned scaffolds were furthermore analyzed for the presence of clustered regularly interspaced short palindromic repeats (CRISPRs) using the CRISPR PILER-CR v1.026.

The annotated Olavius spp. symbionts' bins were incorporated into the metagenomics version of the U.S. Department of Energy Joint Genome Institute Integrated Microbial Genomes (IMG) ${ }^{27}$, IMG/M (http://img.jgi.doe.gov/m), a data management and analysis platform for metagenomic data. This facilitates access and visualization and comparative analyses of the data in the context of other metagenomic datasets and all publicly available complete microbial genomes.

\section{Community heterogeneity}

To assess nucleotide sequence variation within the binned scaffolds or bins, we analyzed the multiple alignment of the JAZZ assembly. A site was considered polymorphic, if at least two reads showed at least two different nucleotides (or gaps) in regions covered by 4-20 reads. Frequencies of polymorphic sites (the total number of polymorphic sites divided by the total number of nucleotide sites at 4-20X read depth) were averaged over all contigs assigned to a given bin.

\section{Nucleotide sequence deposition}

The metagenomic unassembled sequence reads have been deposited into the NCBI trace archive. Assembled sequences from the Olavius spp. symbionts' metagenome have been deposited into the NCBI database under the project accession AASZ00000000. 
2. Supplementary Figures and Legends

a

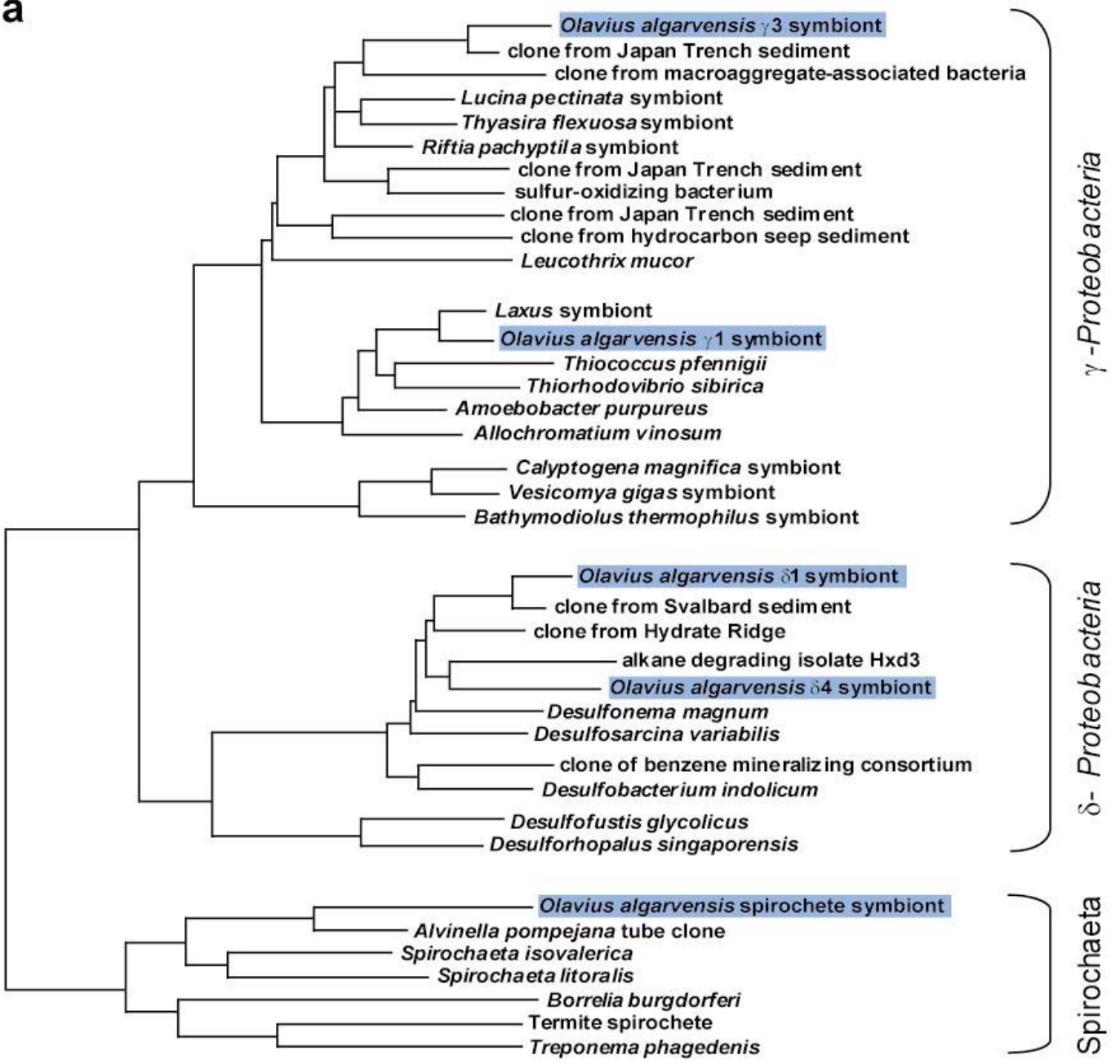

0.10 


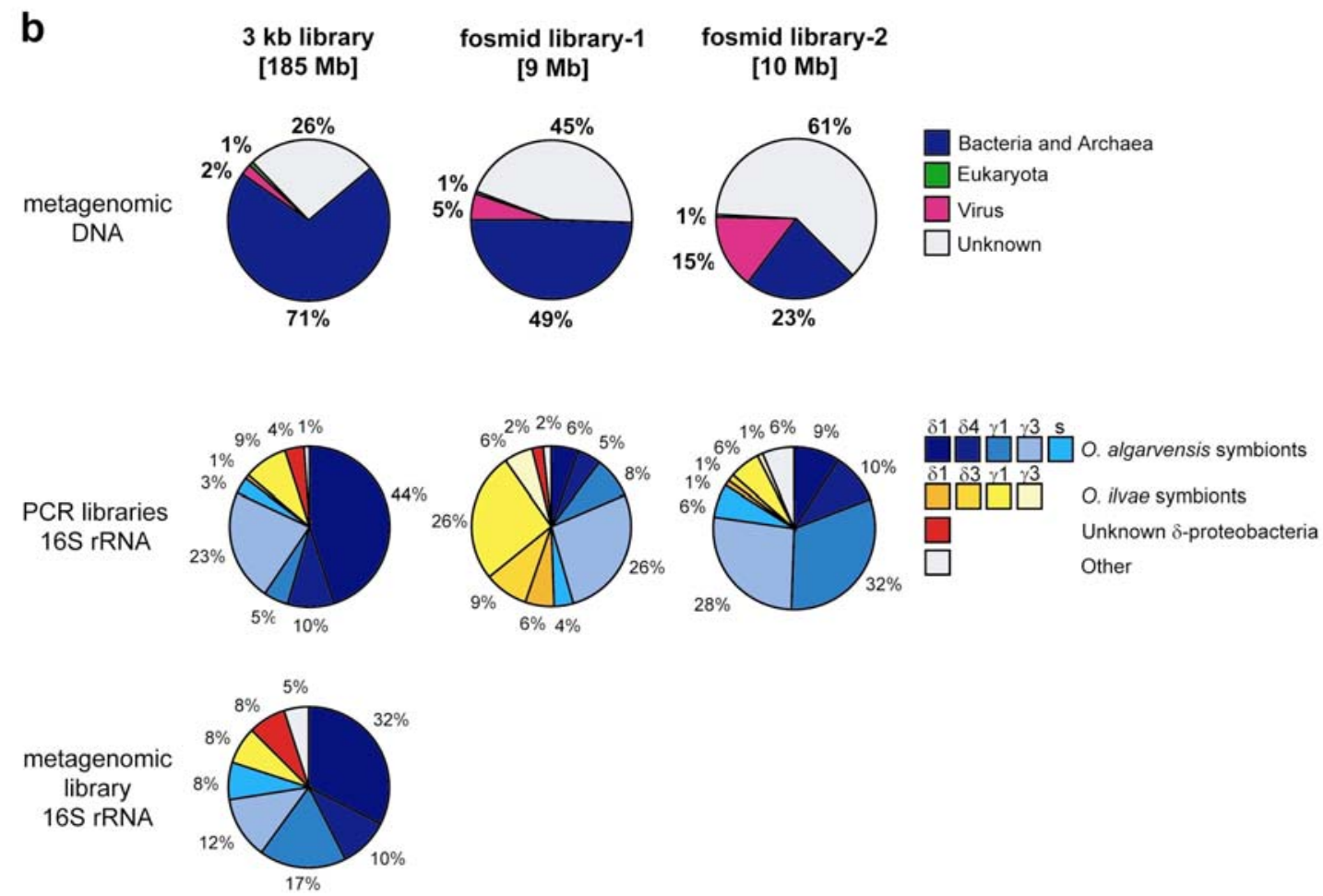

Supplementary Figure S1. (a) Phylogenetic relationship of the $O$. algarvensis endosymbionts (blue) based on parsimony analyses of 16S rRNA sequences. Bar, 10\% estimated sequence divergence. (b) Characterization of the metagenomic libraries and the 16S rRNA PCR libraries. Percentage of $3 \mathrm{~kb}$ and fosmid library end reads (unassembled) with similarities to proteins of bacterial and archaeal, eukaryotic or viral origin (BLASTx, e-value 1e-3, NCBI nr) (top chart row). Unknown = reads with no similarity to proteins in the public databases. The middle chart row indicates the relative phylotype abundance in the DNA used for each library based on 16S rRNA PCR library sequences. For both fosmid libraries this phylotype comparison is a good indication of the natural bacterial abundance, as the DNA originated from pooled worms that were not density fractionated as were those for the $3 \mathrm{~kb}$ library. $\mathrm{s}=$ spirochete. Other includes 16S rRNA phylotypes of low abundance species. The relative phylotype abundance based on 16S rRNA gene representation within the metagenomic $3 \mathrm{~kb}$ library reads is shown in the bottom pie chart. 

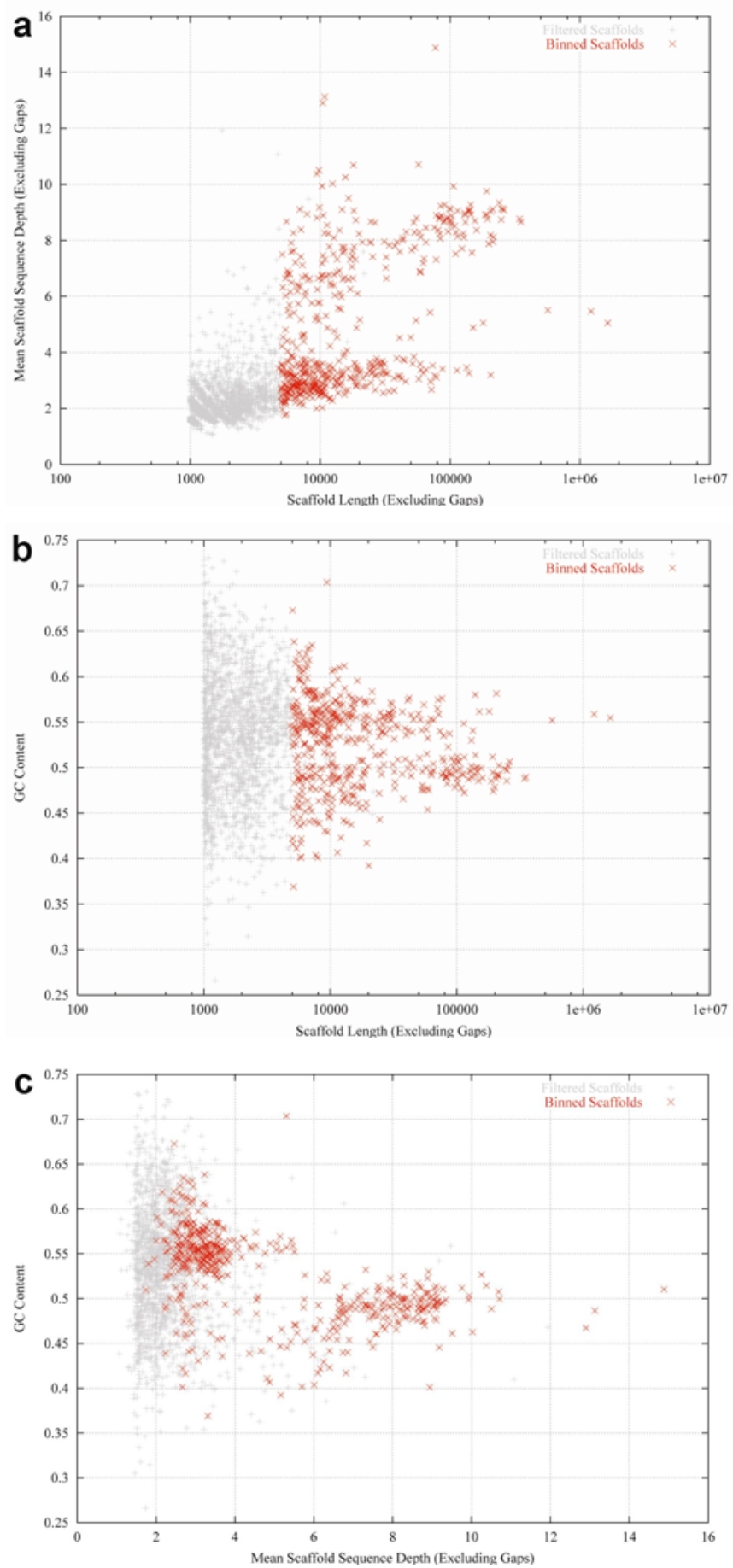

Supplementary Figure S2. Length, sequence depth and GC content distributions of the JAZZ assembled, filtered scaffold set. (a) Net scaffold length vs. mean scaffold sequence depth (excluding gaps). (b) Net scaffold length vs. GC content. (c) Mean scaffold sequence depth (excluding gaps) vs. GC content. The filtered set comprises 2,286 scaffolds with a combined net length of $23.7 \mathrm{Mb}$. The 511 scaffolds that were binned based on nucleotide signatures are shown in red (comprising a net length of $20.1 \mathrm{Mb}$ ). 


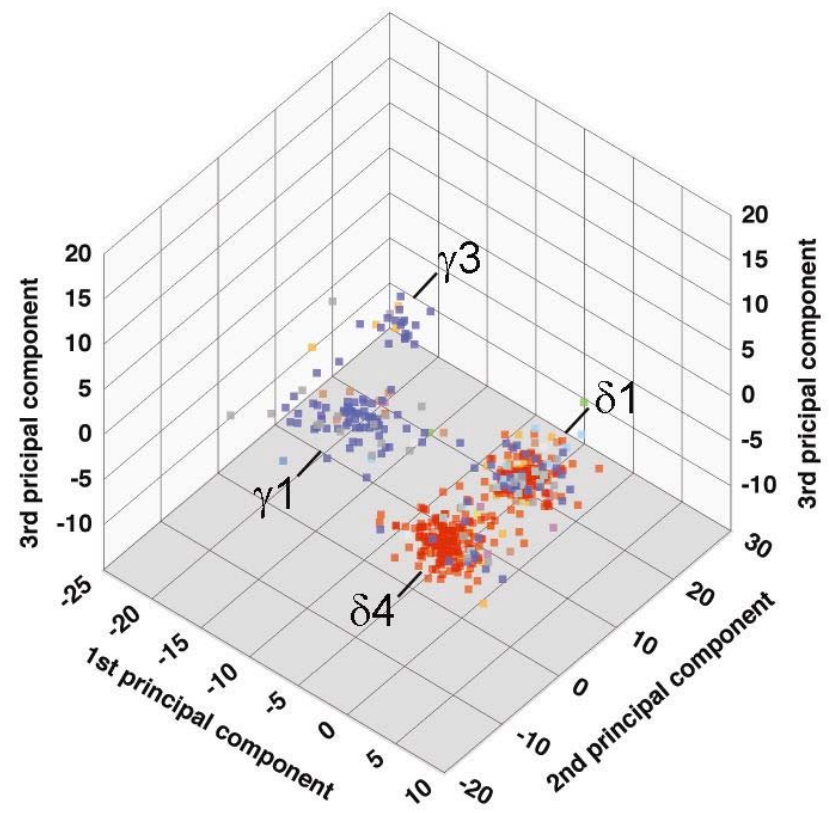

No or ambiguous BLAST hits Acidobacteria Alphaproteobacteria Archaea Bacteroidetes Betaproteobacteria Proteobacteria Chlorobi Chloroflexi Cyanobacteria Deltaproteobacteria Firmicutes Gammaproteobacteria

\section{Supplementary Figure S3. Phylogenetic scaffold affiliations within the nucleotide signature} based symbiont clusters. Visualization of the first three components of a principal component analysis (PCA), in which GC-content, net read depth, z-scores for all possible 64 trinucleotides and 256 tetranucleotides were incorporated with equal weight (z-scores calculated with TETRA and normalized by length); sequences $<5 \mathrm{~kb}$ are not represented. Phylogenetic affiliation of each scaffold was based on the most common phylogeny of its predicted proteins and is indicated by color. 


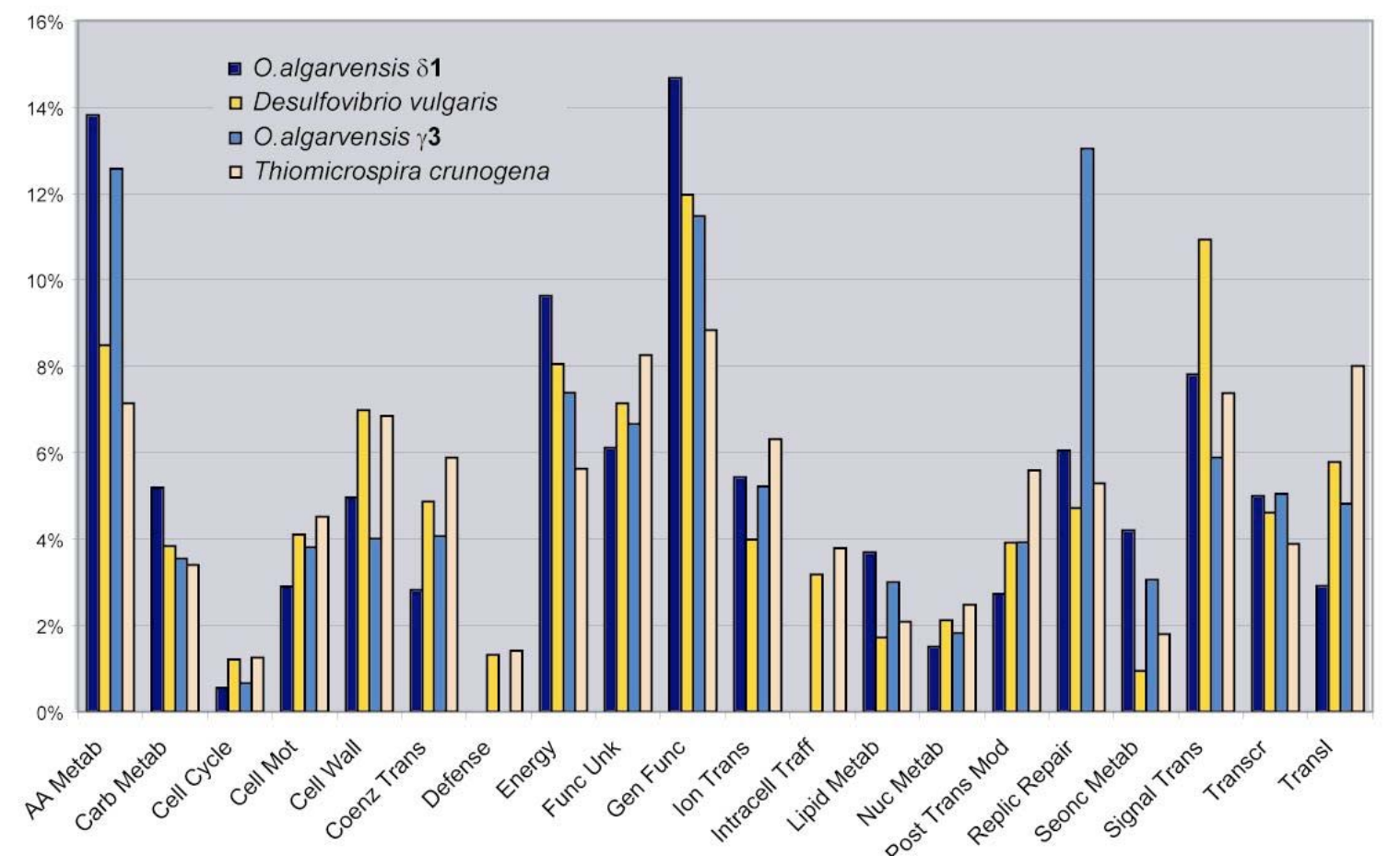

Supplementary Figure S4. Distribution of the genes identified for $\delta 1$ and $\gamma 3$ bins by broad functional category of clusters of orthologous groups of proteins (COGs) (e-value 1e-5), as compared to the complete genomes of Desulfovibrio vulgaris Hildenborough and Thiomicrospira crunogena XCL-2. Categories which show gene representations below $0.2 \%$ are excluded. Both symbiont genome bins show a higher incidence of genes involved in amino acid as well as lipid transport and metabolism as compared to the non-symbiotic bacteria, while genes involved in translation, ribosomal structure and biogenesis show a lower relative abundance. Genes involved in replication, recombination and repair are furthermore highly represented within the $\gamma 3$ bin. 
3. Supplementary Tables

\begin{tabular}{|c|c|c|c|c|}
\hline \multicolumn{2}{|c|}{ Supplementary Table S1. General features of the } & \multirow{2}{*}{$\begin{array}{c}\text { O. algarvensis } \\
\delta_{4}\end{array}$} & \multicolumn{2}{|l|}{ symbiont bins } \\
\hline & $\delta_{1}$ & & $\gamma_{1}$ & $\gamma_{3}$ \\
\hline \multicolumn{5}{|l|}{ Assembly statistics } \\
\hline Genome bin size [bp] & $13,536,737$ & $6,382,161$ & $5,317,000$ & $4,647,793$ \\
\hline Gaps filled with N's [\%] & 16 & 52 & 73 & 12 \\
\hline Number of scaffolds & 226 & 172 & 91 & 22 \\
\hline GC content [\%] & 49.2 & 54.6 & 57.5 & 55.7 \\
\hline Mean total read depth* & 7.1 & 1.6 & 0.8 & 4.6 \\
\hline Mean net read depth* & 8.4 & 3.3 & 3.0 & 5.2 \\
\hline Mean total length [bp] & 59,897 & 37,106 & 58,429 & 211,263 \\
\hline Mean net length [bp] & 50,593 & 17,887 & 16,059 & 185,783 \\
\hline \multicolumn{5}{|l|}{ Gene predictions } \\
\hline Protein coding genes & 12,084 & 3,012 & 1,872 & 4,154 \\
\hline Genes with similarity to $\mathrm{nr}$ & 7,505 & 2,399 & 1,302 & 3,778 \\
\hline Genes with similarity to COG & 5,340 & 1,919 & 831 & 3,083 \\
\hline Number of rRNA operons & 1 & 1 & $2^{\star *}$ & 1 \\
\hline Number of tRNA genes & 49 & 23 & 17 & 33 \\
\hline Number of tRNA synthetases & 26 & 22 & 12 & 26 \\
\hline
\end{tabular}

*normalized with respect to length.

**one complete rRNA operon and one partial 16S rRNA gene.

nr. non-redundant Genbank. 
of other extracellular as well as intracellular endosymbionts

\begin{tabular}{|c|c|c|c|c|c|c|}
\hline endosymbiont (class) & host organism & $\begin{array}{c}\text { Genome } \\
\text { (bin) } \\
\text { size* }^{*} \\
\text { [Mb] }\end{array}$ & $\begin{array}{c}\text { GC } \\
\text { content } \\
{[\%]}\end{array}$ & $\begin{array}{c}\text { Number } \\
\text { of } \\
\text { protein } \\
\text { coding } \\
\text { genes }\end{array}$ & $\begin{array}{l}\text { Number } \\
\text { of rRNA } \\
\text { operons }\end{array}$ & $\begin{array}{c}\text { Number } \\
\text { of tRNA } \\
\text { genes }\end{array}$ \\
\hline O.algarvensis $\quad \partial_{1}$ symbiont $\left(\delta_{-}\right.$Proteob. $)$ & annelid O.algarvensis & 13.5 & 49.2 & 12,084 & 1 & 49 \\
\hline O.algarvensis $\quad \mathrm{d}_{4}$ symbiont( $\quad \mathrm{d}_{\text {-Proteob. }}$ ) & annelid O.algarvensis & 6.4 & 54.6 & 3,012 & 1 & 23 \\
\hline O.algarvensis $\quad \gamma_{1}$ symbiont $(\quad \gamma$-Proteob.) & annelid O.algarvensis & 5.3 & 57.5 & 1,872 & $2^{* *}$ & 17 \\
\hline O.algarvensis $\left.\gamma_{3 \text { symbiont }} \quad \gamma_{\text {-Proteob. }}\right)$ & annelid O.algarvensis & 4.6 & 55.7 & 4,154 & 1 & 33 \\
\hline Photorhabdus luminescens $\quad{ }^{28}$ ( $\gamma$-Proteob.) & nematodes (Heterorhabditidae) & 5.7 & 42.8 & 4,839 & 7 & 85 \\
\hline Vibrio fischeri $\mathrm{ES}_{114}{ }^{29}$ ( $\gamma$-Proteob.) & bobtail squid Euprymnascolopes & 4.3 & 38.3 & 3,647 & 12 & 119 \\
\hline Bradyrhizobium japonicum $\quad{ }^{30}(\alpha-\text {-Proteob. })^{\star \star \star}$ & soybean Glycinemax & 9.1 & 64 & 8,317 & 1 & 50 \\
\hline Buchnera aphidicola ${ }^{31}(\gamma \text {-Proteob. })^{\star \star *}$ & aphid Baizongiapistacea & 0.6 & 25 & 504 & 1 & 32 \\
\hline
\end{tabular}

*induding plasmids.

**one complete IRNA operon and one partial 16S IRNAgene.

*** intracellularendosymbiontlocation. 


\begin{tabular}{|c|c|c|c|c|}
\hline \multicolumn{3}{|c|}{$\begin{array}{l}\text { Supplementary Table S3. Amino acyl tRNA synthetase genes in the } \\
\text { symbiont bins }\end{array}$} & \multicolumn{2}{|c|}{ O. algarvensis } \\
\hline & $\delta_{1}$ & $\delta_{4}$ & $\gamma_{1}$ & $\gamma 3$ \\
\hline Glutamyl- and glutaminyl-tRNA synthetase & 1 & 0 & 3 & 3 \\
\hline Alanyl--tRNA synthetase & 1 & 1 & 0 & 1 \\
\hline Phenylalanyl-tRNA synthetase & 1 & 1 & 0 & 1 \\
\hline Asparty/lasparaginyl-tRNA synthetase & 1 & 0 & 0 & 0 \\
\hline Arginyl-tRNA synthetase & 1 & 1 & 1 & 1 \\
\hline Isoleucyl-tRNA synthetase & 1 & 1 & 0 & 1 \\
\hline Phenylalanyl-tRNA synthetase beta subunit & 1 & 1 & 0 & 2 \\
\hline Histidyl-tRNA synthetase & 1 & 0 & 1 & 2 \\
\hline Tyrosyl-tRNA synthetase & 0 & 0 & 0 & 1 \\
\hline Seryl-tRNA synthetase & 1 & 1 & 0 & 2 \\
\hline Aspartyl-tRNA synthetase & 1 & 1 & 1 & 1 \\
\hline Tryptophanyl-tRNA synthetase & 1 & 0 & 1 & 0 \\
\hline Cysteinyl-tRNA synthetase & 1 & 3 & 0 & 1 \\
\hline Threonyl-tRNA synthetase & 2 & 1 & 0 & 1 \\
\hline Prolyl-tRNA synthetase & 1 & 1 & 1 & 1 \\
\hline Leucyl-tRNA synthetase & 1 & 1 & 2 & 1 \\
\hline Valyl-tRNA synthetase & 1 & 2 & 0 & 1 \\
\hline Glycyl-tRNA synthetase beta subunit & 1 & 0 & 0 & 2 \\
\hline Glycyl-tRNA synthetase alpha subunit & 1 & 0 & 0 & 1 \\
\hline Lysyl-tRNA synthetase class II & 2 & 2 & 0 & 2 \\
\hline Lysyl-tRNA synthetase class I & 0 & 0 & 0 & 0 \\
\hline Pseudouridine-tRNA synthetase & 3 & 2 & 0 & 1 \\
\hline Methionyl-tRNA synthetase & 2 & 2 & 1 & 0 \\
\hline tRNA-dihydrouridine synthetase & 0 & 1 & 0 & 0 \\
\hline tRNA(Ile)-lysidine synthetase & 0 & 0 & 1 & 0 \\
\hline
\end{tabular}




\begin{tabular}{|c|c|c|c|c|}
\hline \multicolumn{3}{|c|}{$\begin{array}{l}\text { Supplementary Table S4. Repeats and polymorphic sites in the } \\
\text { symbiont bins }\end{array}$} & \multicolumn{2}{|c|}{ O. algarvensis } \\
\hline & $\delta_{1}$ & $\delta_{4}$ & $\gamma_{1}$ & $\gamma_{3}$ \\
\hline Number of transposases & 276 & 17 & 389 & 313 \\
\hline Percent of transposases & 2.3 & 0.6 & 20.5 & 7.5 \\
\hline Number of integrases & 30 & 3 & 28 & 78 \\
\hline CRISPR elements & yes & no & no & no \\
\hline Number of polymorphic sites* & 7565 & 144 & 422 & 1280 \\
\hline Frequencies of polymorphic sites ${ }^{\star *}[\%]$ & 0.08 & 0.01 & 0.1 & 0.04 \\
\hline
\end{tabular}

*a site was considered polymorphic, if at least two reads showed at least two different nucleotides (or gaps) in regions covered by 4-20 reads.

**averaged over all contigs assigned to a given bin. 
Supplementary TableS5. Amino acid and vitamin biosynthesis genes in the

O.algarvensis symbiont bins

$\begin{array}{llll}\delta 1 & \delta 4 & \gamma 1 & \gamma 3\end{array}$

amino acid biosynthesis

histidine

phenylalanine

tyrosine

leucine

isoleucine

valine

tryptophan

arginine

lysine

methionine

threonine

serine

proline

glycine

cycteine

asparagine

glutamine

alanine

aspartic acid

glutamic acid

selenocysteine

pyrrolysine

coenzymes and cofactor biosynthesis

biotin

cobalamin

coenzyme A

riboflavin and $F A D$

heme

NAD

pyridoxal phosphate

thiamin

ubiquinone

$\begin{array}{llll}+ & + & \pm & + \\ + & + & \pm & + \\ + & + & \pm & + \\ + & + & \pm & + \\ + & + & \pm & + \\ + & + & \pm & + \\ + & + & - & + \\ + & + & \pm & + \\ + & + & \pm & + \\ + & + & \pm & + \\ \pm & + & \pm & \pm \\ + & + & \pm & + \\ + & + & \pm & + \\ + & + & \pm & + \\ + & + & \pm & \pm \\ + & + & \pm & \pm \\ + & + & + & + \\ + & + & + & + \\ + & + & - & + \\ + & + & - & + \\ + & + & - & + \\ + & + & +\end{array}$

+ at least $80 \%$ of the renu lired sunthesis nenes are nresent 


\begin{tabular}{|c|c|c|c|c|}
\hline \multicolumn{2}{|c|}{$\begin{array}{l}\text { Supplementary Table S6. Transposase genes in the } \\
\text { symbiont bins }\end{array}$} & \multicolumn{2}{|c|}{ O.algarvensis } & \multirow[b]{2}{*}{$\gamma 3$} \\
\hline & $\delta_{1}$ & $\delta_{4}$ & $\gamma_{1}$ & \\
\hline IS1 & - & - & 48 & - \\
\hline IS106 & - & - & 3 & - \\
\hline IS110 & - & - & 8 & - \\
\hline IS111A & - & - & 3 & - \\
\hline IS111A/S1328/S1533 & - & 1 & - & - \\
\hline IS116 & - & - & 5 & - \\
\hline IS116/S110/S902 & - & 1 & - & - \\
\hline IS1249 & 10 & - & - & - \\
\hline IS1479 & - & - & - & 1 \\
\hline IS1480 & - & - & 1 & - \\
\hline IS1595 & 1 & - & - & - \\
\hline IS1663 & - & - & 1 & - \\
\hline IS180 & - & - & 3 & - \\
\hline IS186 & 13 & - & - & - \\
\hline IS200 & - & - & 5 & 2 \\
\hline IS21 & 2 & - & - & - \\
\hline IS298 & - & - & 1 & - \\
\hline IS3 & - & - & - & 4 \\
\hline IS3/S911 & 1 & - & 12 & 59 \\
\hline IS3231 & 28 & - & - & - \\
\hline IS4 & 60 & - & 52 & 77 \\
\hline IS5 & - & - & 4 & - \\
\hline IS630 & 1 & - & 73 & - \\
\hline IS641 & - & - & - & 27 \\
\hline IS642 & - & - & 2 & - \\
\hline IS643 & 1 & - & - & - \\
\hline IS653 & 2 & - & - & - \\
\hline IS66 & 1 & - & - & 9 \\
\hline ISChy4 & 2 & - & - & - \\
\hline ISCps6 & - & - & - & 1 \\
\hline ISDvu4 & 2 & 6 & - & - \\
\hline ISEcp1 & - & - & 13 & - \\
\hline ISGsu4 & 5 & - & - & 7 \\
\hline ISGsu6 & 1 & - & - & - \\
\hline Iso-IS1 & - & - & 20 & - \\
\hline ISPpu8 & 3 & - & - & - \\
\hline ISPsy19 & - & - & 1 & - \\
\hline ISPsy5 & - & - & - & 1 \\
\hline ISR013 & 5 & - & - & - \\
\hline ISRm & - & - & 4 & - \\
\hline ISRm22 & - & - & - & 18 \\
\hline ISRPsy14 & 5 & - & - & - \\
\hline ISSod13 & 5 & 8 & - & - \\
\hline ISSpo2 & - & - & 1 & - \\
\hline ISSpo3 & 1 & - & - & - \\
\hline ISSpo8 & 99 & - & - & - \\
\hline ISxac3 & 1 & - & - & - \\
\hline Tnp & 3 & - & - & - \\
\hline TnpA & - & - & 16 & 2 \\
\hline Transposase & 10 & - & 75 & 13 \\
\hline Transnnsase \& inactiva & - & 1 & 19 & 81 \\
\hline
\end{tabular}




\section{Supplementary Discussion}

\section{Sample characterization}

O. algarvensis specimens were collected from shallow subtidal sediments off the island of Elba, Italy. O. algarvensis is the dominant gutless oligochaete species at the collection site, where a second gutless oligochaete species, O. ilvae co-occurs in low abundance ${ }^{1}$. Shotgun libraries were constructed from pooled fresh endosymbiont enriched sample (3 $\mathrm{kb}$ library), as well as pooled frozen worm specimens (fosmid libraries), due to the unavailability of large amounts of fresh sample. We generated 185 million bases $(\mathrm{Mb})$ of $3 \mathrm{~kb}$ library end sequence and $19 \mathrm{Mb}$ of fosmid end sequence, for a total of $204 \mathrm{Mb}$ of high-quality shotgun sequence data. BLAST analysis ${ }^{5}$ of the unassembled sequence reads as well as $16 \mathrm{~S}$ rRNA gene analysis indicated that the $3 \mathrm{~kb}$ library largely consists of $O$. algarvensis symbiont DNA, supporting our choice of this library for in-depth sequencing (Supplementary Fig. S1b).

To estimate the relative abundance of the $O$. algarvensis symbionts within the libraries, we performed 16S rRNA PCR amplification of each source DNA using parallel PCR reaction aliquots, low PCR cycle numbers and a reconditioning step to minimize

PCR bias as well as chimera and heteroduplex formation ${ }^{3}$. The 16S rRNA gene analysis of the DNA source used for the $3 \mathrm{~kb}$ library construction revealed that sequences derived from the $O$. algarvensis symbionts were highly represented (Supplementary Fig. S1b), dominated by $\delta 1$ and $\gamma 3$. Additional 16S rRNA sequences derived from an unknown Deltaproteobacteria closely related to $O$. algarvensis $\delta 1$, O. ilvae $\gamma 1$ and other bacterial species at low abundance. 16S rRNA gene sequences found within the metagenomic $3 \mathrm{~kb}$ library showed a similar distribution of phylotypes (Supplementary Fig. S1b). 16S rRNA gene analyses of DNA used for the fosmid libraries showed that all three samples differed in their symbiont species distribution and in contamination with $O$. ilvae symbionts (Supplementary Fig. S1b). This may be due to variations in oligochaete species distribution patterns. 


\section{Metagenomic data analysis and binning}

The metagenomic shotgun sequences were assembled using the whole-genome shotgun assembler $\mathrm{JAZZ}^{8}$, resulting in a total of 2,286 scaffolds with a combined total length of $39.3 \mathrm{Mb}$ (net length not including gaps was $23.7 \mathrm{Mb}$; Supplementary Fig. S2) and a longest scaffold of $1.9 \mathrm{Mb}$ (total length). Binning of the Olavius spp. symbionts' metagenome resulted in 511 scaffolds (Supplementary Fig. S2) forming four distinct clusters, identified as $O$. algarvensis symbionts $\delta 1, \delta 4, \gamma 1$, and $\gamma 3$, as based on 16S rRNA genes. Symbiont bin assignments were supported by phylogenetic analysis of predicted proteins within each cluster of scaffolds the distribution of 49 single-copy genes. Only one gene, secG encoding the preprotein translocase $\mathrm{SecG}$ subunit, was found in duplicate and only in the $\delta 1$ bin (sequence similarities between the two copies of $\sec G$ were $95 \%$ at the nucleotide level and $98 \%$ at the amino acid level). This could indicate the presence of more than one strain in this bin and might explain its large size of $13.5 \mathrm{Mb}$ (total length), although genomes of comparable size are known from the Deltaproteobacteria (e.g. Polyangium cellulosum) (http://genomesonline.org) ${ }^{32}$. The single occurrence of 48 out of 49 single-copy genes in the $\delta 1$ bin and the presence in single copy of other key genes, such as most ribosomal proteins, cell division genes, flagellum genes and amino acyl tRNA synthetases (Supplementary Table S3), is however indicative of the presence of a single dominant strain.

\section{Carbon fixation in the $\delta$-symbionts}

Carbon fixation within both deltaproteobacterial symbionts is possible via the reductive acetyl-CoenzymeA (CoA) pathway and also likely via the reductive tricarboxylic acid (TCA) cycle. Both bins encode citrate lyase and oxoglutarate-ferrodoxin oxidoreductase, which catalyze two out of three potentially irreversible steps in the TCA. The third potentially irreversible step is catalyzed by succinate dehydrogenase (SDH). Depending on the specific implementation of this enzyme, it could be either reversible (as in Bacillus subtilis) or irreversible (as in E. coli, which has a separate succinate dehydrogenase and fumarate reductase). Succinate dehydrogenase is encoded in both proteobacterial symbionts, yet we are unable to determine in which direction their SDH catalyzes its reaction and whether it is reversible. The proteins highest percent identity is to the 
actinobacterial succinate dehydrogenase. In actinobacteria, this enzyme strictly catalyzes succinate oxidation, while a second enzyme catalyzes fumarate reduction. However, in general the direction of reaction of SDH/QFR is dependent on the structure and type of cytochrome subunit; yet we were unable to find any cytochrome subunit in deltaproteobacterial bins (and no hydrophobic anchor subunit for that matter). The lack of subunit may either be due to the incompleteness of these genome bins, or the symbionts have a very unusual form of this enzyme, a soluble form. A soluble fumarate reductase is known in methanogenic archaea, where a soluble donor (coenzyme M) is used instead of a quinone.

\section{Osmolyte breakdown}

Bacterial $^{33}$ and archaeal ${ }^{34}$ TMAO breakdown pathways are present in the $O$. algarvensis symbionts: several homologs of trimethylamine/dimethylamine dehydrogenase are found in the $\gamma 3$ and $\delta 1$ symbiont bins and 22 proteins from the trimethylamine:corrinoid methyltransferase family were encoded in $\gamma 3, \delta 1$ and $\delta 4$ symbionts, as well as on unassigned scaffolds. Six genes coding for dimethylamine:corrinoid methyltransferase and two genes encoding monomethylamine:corrinoid methyltransferase are present in the $\delta 1$ bin. Like their archaeal counterparts, at least two proteins from the $\delta 1$ symbiont coding for trimethylamine methyltransferases and all proteins encoding dimethylamine methyltransferases are interrupted by an amber stop codon UAG, which is most likely translated to pyrrolysine. This is supported by the presence of pyrrolysine-specific aminoacyl-tRNA synthetase and PylS-associated genes in the $\delta 1$ symbiont. Tetrahydrofolate appears to be the most likely acceptor of methyl groups in the symbionts due to the presence of at least three homologs of methylcobalamin:tetrahydrofolate methyltransferase MtvA from the vanillate demethylase complex of Moorella thermoacetica ${ }^{35}$. Some of the methylamine methyltransferase genes are found in chromosomal clusters with the genes encoding putative enzymes from the glycine oxidase/dimethylglycine dehydrogenase family and the molybdopterin dehydrogenase family, suggesting the existence of branched and possibly novel pathways for degradation of trimethylamine $\mathrm{N}$-oxide. 


\section{Secondary metabolites}

Many marine invertebrates are associated with endo- and epibiotic microorganisms producing secondary metabolites of large molecular diversity, which led us to evaluate the symbiont bins for genes indicative for the biosynthesis of such compounds. Both deltaproteobacterial bins encode genes likely involved in polyketide and non-ribosomal peptide synthesis, including non-ribosomal peptide synthases, polyketide synthases, and methyltransferases. The products of these genes could be toxins and antibiotics, used possibly by the hosts as a protection against predation, or signaling molecules involved in symbiont - host interactions.

None of the symbiont genome bins were found to encode any of the key enzymes involved in the biosynthesis of steroid hormones and catecholamines (dopamine, norepinephrine, epinephrine). Homoserine lactone synthases, involved in quorum sensing, were also not encoded in any of the symbionts. It is not possible to decipher which compounds produced by the symbionts could have an effect on host behavior based on gene content alone.

\section{Symbiont transmission}

Symbiotic bacteria without a free-living stage are transmitted vertically from one generation to the next. In gutless oligochaetes, at least some of the symbionts may be transmitted vertically in a smear-like infection as the eggs exit the worm and pass genital pads packed with the symbiotic bacteria ${ }^{36}$. However, the deposition of the eggs directly into the surrounding sediments would also offer free-living bacteria from the environment an opportunity to invade the egg. It is therefore possible that some of the symbionts are inherited vertically from the parents and some horizontally from the environment. It is also possible that the same symbiont is transmitted vertically as a rule, but can also be acquired from the environment as shown for the Wolbachia symbionts in $\operatorname{arthropods}^{37,38}$. The low levels of polymorphism in all four symbiont bins of $O$. algarvensis do not exclude horizontal transmission, as selection by the host for a single bacterial strain is well known from other marine symbioses in which the symbionts are acquired from the environment, such as the luminescence symbioses between squid and Vibrio fischeri ${ }^{39,40}$. 


\section{Supplementary References}

1. Giere, O. \& Erseus, C. Taxonomy and new bacterial symbioses of gutless marine Tubificidae (Annelida, Oligochaete) from the Island of Elba (Italy). Org. Divers. Evol. 2, 289-297 (2002).

2. Lane, D. J. 16S/23S rRNA sequencing. In Nucleic Acid Techniques in Bacterial Systematics (eds. Stachebrandt, E. \& Goodfellow, M.) (Wiley, Chichester, New York, 1991).

3. Thompson, J. R., Marcelino, L. A. \& Polz, M. F. Heteroduplexes in mixedtemplate amplifications: formation, consequence and elimination by 'reconditioning PCR'. Nucleic Acids Res. 30, 2083-8 (2002).

4. Huber, T., Faulkner, G. \& Hugenholtz, P. Bellerophon: a program to detect chimeric sequences in multiple sequence alignments. Bioinformatics 20, 2317-9 (2004).

5. Altschul, S. F., Gish, W., Miller, W., Myers, E. W. \& Lipman, D. J. Basic local alignment search tool. J. Mol. Biol. 215, 403-10 (1990).

6. Ludwig, W. et al. ARB: a software environment for sequence data. Nucleic Acids Res. 32, 1363-71 (2004).

7. Chapman, J., Putnam, N., Ho, I. \& Rokhsar, D. JAZZ, a whole genome shotgun assembler. Unpublished.

8. Aparicio, S. et al. Whole-genome shotgun assembly and analysis of the genome of Fugu rubripes. Science 297, 1301-10 (2002).

9. Kent, W. J. BLAT--the BLAST-like alignment tool. Genome Res. 12, 656-64 (2002).

10. Karlin, S. \& Burge, C. Dinucleotide relative abundance extremes: a genomic signature. Trends Genet. 11, 283-90 (1995).

11. Teeling, H., Meyerdierks, A., Bauer, M., Amann, R. \& Glockner, F. O. Application of tetranucleotide frequencies for the assignment of genomic fragments. Environ. Microbiol. 6, 938-47 (2004).

12. Deschavanne, P. J., Giron, A., Vilain, J., Fagot, G. \& Fertil, B. Genomic signature: characterization and classification of species assessed by chaos game representation of sequences. Mol. Biol. Evol. 16, 1391-9 (1999).

13. Wang, Y., Hill, K., Singh, S. \& Kari, L. The spectrum of genomic signatures: from dinucleotides to chaos game representation. Gene 346, 173-85 (2005).

14. Huntemann, M. MetaClust - Entwicklung eines modularen Programms zum Clustern von Metagenomfragmenten anhand verschiedener intrinsischer DNASignaturen. Thesis, University Bremen (2006).

15. de Hoon, M. J., Imoto, S., Nolan, J. \& Miyano, S. Open source clustering software. Bioinformatics 20, 1453-4 (2004).

16. Sandberg, R. et al. Capturing whole-genome characteristics in short sequences using a naive Bayesian classifier. Genome Res. 11, 1404-9 (2001).

17. Badger, J. H. \& Olsen, G. J. CRITICA: coding region identification tool invoking comparative analysis. Mol. Biol. Evol. 16, 512-24 (1999). 
18. Delcher, A. L., Harmon, D., Kasif, S., White, O. \& Salzberg, S. L. Improved microbial gene identification with GLIMMER. Nucleic Acids Res. 27, 4636-41 (1999).

19. Guo, F. B., Ou, H. Y. \& Zhang, C. T. ZCURVE: a new system for recognizing protein-coding genes in bacterial and archaeal genomes. Nucleic Acids Res. 31, 1780-9 (2003).

20. Bendtsen, J. D., Nielsen, H., von Heijne, G. \& Brunak, S. Improved prediction of signal peptides: SignalP 3.0. J. Mol. Biol. 340, 783-95 (2004).

21. Krogh, A., Larsson, B., von Heijne, G. \& Sonnhammer, E. L. Predicting transmembrane protein topology with a hidden Markov model: application to complete genomes. J. Mol. Biol. 305, 567-80 (2001).

22. Meyer, F. et al. GenDB--an open source genome annotation system for prokaryote genomes. Nucleic Acids Res. 31, 2187-95 (2003).

23. Lowe, T. M. \& Eddy, S. R. tRNAscan-SE: a program for improved detection of transfer RNA genes in genomic sequence. Nucleic Acids Res. 25, 955-64 (1997).

24. Quast, C. MicHanThi - Design and Implementation of a System for the Prediction of Gene Functions in Genome Annotation Projects. Thesis, University Bremen (2006).

25. Tatusov, R. L. et al. The COG database: an updated version includes eukaryotes. BMC Bioinformatics 4, 41 (2003).

26. Edgar, R. C. \& Myers, E. W. PILER: identification and classification of genomic repeats. Bioinformatics 21 Suppl 1, i152-i158 (2005).

27. Markowitz, V. M. et al. The integrated microbial genomes (IMG) system. Nucleic Acids Res. 34, D344-8 (2006).

28. Duchaud, E. et al. The genome sequence of the entomopathogenic bacterium Photorhabdus luminescens. Nat. Biotechnol. 21, 1307-13 (2003).

29. Ruby, E. G. et al. Complete genome sequence of Vibrio fischeri: a symbiotic bacterium with pathogenic congeners. Proc. Natl Acad. Sci. U. S. A. 102, 3004-9 (2005).

30. Kaneko, T. et al. Complete genomic sequence of nitrogen-fixing symbiotic bacterium Bradyrhizobium japonicum USDA110. DNA Res. 9, 189-97 (2002).

31. van Ham, R. C. et al. Reductive genome evolution in Buchnera aphidicola. Proc. Natl Acad. Sci. U. S. A. 100, 581-6 (2003).

32. Liolios, K., Tavernarakis, N., Hugenholtz, P. \& Kyrpides, N. C. The Genomes On Line Database (GOLD) v.2: a monitor of genome projects worldwide. Nucleic Acids Res. 34, D332-4 (2006).

33. Yang, C. C., Packman, L. C. \& Scrutton, N. S. The primary structure of Hyphomicrobium $\mathrm{X}$ dimethylamine dehydrogenase. Relationship to trimethylamine dehydrogenase and implications for substrate recognition. Eur. J. Biochem. 232, 264-71 (1995).

34. Paul, L., Ferguson, D. J., Jr. \& Krzycki, J. A. The trimethylamine methyltransferase gene and multiple dimethylamine methyltransferase genes of Methanosarcina barkeri contain in-frame and read-through amber codons. $J$. Bacteriol. 182, 2520-9 (2000).

35. Naidu, D. \& Ragsdale, S. W. Characterization of a three-component vanillate Odemethylase from Moorella thermoacetica. J. Bacteriol. 183, 3276-81 (2001). 
36. Giere, O. \& Langheld, C. Structural organisation, transfer and biological fate of endosymbiotic bacteria in gutless oligochaetes. Mar. Biol. 93, 641-650 (1987).

37. Vavre, F., Fleury, F., Lepetit, D., Fouillet, P. \& Bouletreau, M. Phylogenetic evidence for horizontal transmission of Wolbachia in host-parasitoid associations. Mol. Biol. Evol. 16, 1711-23 (1999).

38. Huigens, M. E., de Almeida, R. P., Boons, P. A., Luck, R. F. \& Stouthamer, R. Natural interspecific and intraspecific horizontal transfer of parthenogenesisinducing Wolbachia in Trichogramma wasps. Proc. Biol. Sci. 271, 509-15 (2004).

39. Nishiguchi, M. K., Ruby, E. G. \& McFall-Ngai, M. J. Competitive dominance among strains of luminous bacteria provides an unusual form of evidence for parallel evolution in Sepiolid squid-vibrio symbioses. Appl. Environ. Microbiol. 64, 3209-13 (1998).

40. Visick, K. L. \& McFall-Ngai, M. J. An exclusive contract: specificity in the Vibrio fischeri-Euprymna scolopes partnership. J. Bacteriol. 182, 1779-87 (2000). 